\title{
Some aspects of the Hodge conjecture
}

\author{
Claire Voisin
}

CNRS, Institut de Mathématiques de Jussieu

\section{Contents}

$\begin{array}{llr}0 & \text { Introduction } & 1\end{array}$

0.1 Hodge theory . . . . . . . . . . . . . . . . 2

0.2 Hodge classes and the Hodge conjecture . . . . . . . . . . . 4

0.3 Lefschetz theorem on $(1,1)$-classes $\ldots \ldots \ldots \ldots \ldots$

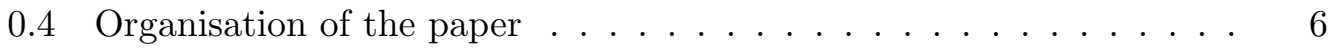

1 The analytic side : Hodge classes and coherent sheaves on Kähler $\begin{array}{ll}\text { manifolds } & 6\end{array}$

1.1 Several constructions of Hodge classes . . . . . . . . . . . . 6

1.2 Weil tori and Weil classes . . . . . . . . . . . . . . . . . 9

1.3 The main result . . . . . . . . . . . . . . . . . . . 11

1.4 Further comments on the $K$-theory of compact Kähler manifolds . . 13

2 The topological side: integral Hodge classes $\quad 14$

2.1 Atiyah-Hirzebruch-Totaro topological obstruction. . . . . . . . . . . 14

2.2 Kollár's example . . . . . . . . . . . . . . . . . . 16

2.3 Rationally connected varieties and the rationality problem . . . . . 17

3 The algebraic side : absolute Hodge classes and Hodge loci 22

3.1 Absolute Hodge classes . . . . . . . . . . . . . . . . . . . 22

3.2 Locus of Hodge classes . . . . . . . . . . . . . . . . . 26

\section{Introduction}

This text is an expanded version of the Takagi lectures I gave at the RIMS, Kyoto, in november 2006. Following my work of the last years around the Hodge conjecture (see [38], [41], and [42]), I decided to insist in these lectures on the fact that, while the Hodge conjecture can be considered as a conjecture either in complex analysis, in differential topology, or in algebraic geometry, the evidences for it are all coming from algebraic geometry.

In fact, the paper [38] shows that, if one extends the geometric setting and consider the case of compact Kähler manifolds, one finds supplementary restrictions for Hodge classes to come from constructions of complex analytic geometry.

On the other hand, in the context of algebraic geometry, one can ask questions concerning the definition fields of the Hodge loci, as the Hodge conjecture predicts 
that they are algebraic and defined on $\overline{\mathbb{Q}}$ (here we note that any complex projective manifold is a member of a family defined over $\mathbb{Q}$ ).

The best evidence for the Hodge conjecture is thus due to Cattani, Deligne and Kaplan [11], who showed that the Hodge loci, and even the connected components of the locus of Hodge classes, are algebraic. We provide in [42] an improvement of their result by giving some criteria for the Hodge loci to be defined over $\overline{\mathbb{Q}}$. We also relate this problem to the question whether the Hodge conjecture can be reduced to the case of varieties defined over $\overline{\mathbb{Q}}$.

We included in the text a section devoted to the case of integral Hodge classes, for which the original Hodge conjecture was disproved by Atiyah and Hirzebruch [4]. We discuss there the possibility of extracting interesting birational invariants from this, using the groups of integral Hodge classes modulo those which are algebraic. This is essentially taken from [41], but the discussion concerning the 4-dimensional case here is new.

Thanks. I thank Yasuyuki Kawahigashi, Toshiyuki Kobayashi, Hiraku Nakajima, Kaoru Ono and Takeshi Saito, the organizers of the first Takagi lectures, for inviting me to deliver these lectures and for giving me the opportunity to write these notes.

\subsection{Hodge theory}

Let us start with the notion of cohomology class of type $(p, q)$ on a complex manifold $X$. On such an $X$, we have the notion of differential form of type $(p, q)$ : these are the complex differential forms $\omega$ (say of class $\mathcal{C}^{\infty}$ ), which can be written in local holomorphic coordinates $z_{1}, \ldots, z_{n}, n=\operatorname{dim}_{\mathbb{C}} X$, and in the multiindex notation:

$$
\omega=\sum_{I, J} \omega_{I, J} d z_{I} \wedge d \bar{z}_{J},|I|=p,|J|=q,
$$

where $\omega_{I, J}$ are $\mathcal{C}^{\infty}$ functions. Let us denote by $A^{p, q}(X)$ the space of $(p, q)$-forms. Thus $A^{p, q}(X) \subset A^{k}(X), p+q=k$, where $A^{k}(X)$ is the space of $C^{\infty}$ complex differential $k$-forms on $X$. Recalling that the complex de Rham cohomology $H^{k}(X, \mathbb{C})$ is defined as

$$
H^{k}(X, \mathbb{C})=\frac{\{\text { closed complex } k-\text { forms on } X\}}{\{\text { exact complex } k-\text { forms on } X\}},
$$

it is natural to define for a complex manifold $X$ and for each $(p, q)$ the space of cohomology classes of type $(p, q)$ by the formula

$$
H^{p, q}(X):=\frac{\{\text { closed complex forms of type }(p, q) \text { on } X\}}{\{\text { exact forms of type }(p, q) \text { on } X\}} .
$$

Each complex differential form of degree $k$ decomposes canonically into its components of type $(p, q)$ because any complex differential form of degree 1 decomposes canonically as a sum of a form of type $(1,0)$ (its $\mathbb{C}$-linear part) and a form of type $(0,1)$ (its $\mathbb{C}$-antilinear part). Unfortunately, the differential $d$ does not preserve this decomposition: in fact, we have

$$
d=\partial+\bar{\partial},
$$

where $\partial: A^{p, q}(X) \rightarrow A^{p+1, q}(X)$, and $\bar{\partial}: A^{p, q}(X) \rightarrow A^{p, q+1}(X)$. For this reason, the notion of class of type $(p, q)$ is not very useful for general complex manifolds. 
First of all, there might be no non zero class of type $(p, q)$, for any $(p, q)$ such that $p+q=k$, while $H^{k}(X, \mathbb{C}) \neq 0$. The standard example is that of a Hopf surface, and $k=1$. Furthermore, it might be the case that a non zero cohomology class could be both of type $(p, q)$ and $\left(p^{\prime}, q^{\prime}\right)$ with $(p, q) \neq\left(p^{\prime}, q^{\prime}\right)$.

These pathologies do not appear in the compact Kähler case. This is a famous result due to Hodge, which comes as a consequence of the theory of harmonic forms, and of the so-called Kähler identities.

A Kähler metric is a Hermitian metric on the tangent bundle of a complex manifold $X$ (which has a natural structure of complex vector bundle), which fits very nicely with the complex structure on $X$. The Hermitian metric $h$ being locally written in holomorphic coordinates as $\sum_{i, j} h_{i j} d z_{i} \otimes d \bar{z}_{j}$, there is the corresponding real $(1,1)$-form

$$
\omega=\frac{i}{2} \sum_{i, j} h_{i j} d z_{i} \wedge d \bar{z}_{j}
$$

(the Kähler form), and the Kähler condition is simply $d \omega=0$.

One good way to see that these metrics fit nicely with the complex structure is the following characterization:

Proposition 1 The metric $h$ on $X$ is Kähler if and only if the operator of complex structure I (defining the complex structure of the tangent bundle of $X$ ) is parallel with respect to the Levi-Civita connection associated to the underlying Riemannian metric $g$.

From our point of view, the importance of the Kähler condition lies in the following theorem, due to Hodge.

Theorem 2 If $(X, \omega)$ is Kähler, the Laplacian $\Delta_{g}=d d^{*}+d^{*} d$ associated to the underlying metric $g$ is bihomogeneous, namely satisfies

$$
\Delta_{g}\left(A^{p, q}(X)\right) \subset A^{p, q}(X) .
$$

Recall that a form $\alpha$ is said to be harmonic if $\Delta_{g} \alpha=0$, or equivalently in the compact case, $d \alpha=d^{*} \alpha=0$.

Corollary 3 A differential form $\alpha$ on a Kähler manifold $X$ is harmonic if and only if its components of type $(p, q)$ are harmonic.

Note that, in the compact case, $\alpha$ and its components $\alpha^{p, q}$ are closed, and this provides a decomposition of the cohomology class of $\alpha$ into classes of type $(p, q)$.

One other remarkable fact, which implies Theorem 2 , is the fact that we have the following relation between the three Laplacians $\Delta_{g}, \Delta_{\partial}:=\partial \partial^{*}+\partial^{*} \partial, \Delta_{\bar{\partial}}:=$ $\overline{\partial \partial}^{*}+\bar{\partial}^{*} \bar{\partial}$ :

$$
\Delta_{g}=2 \Delta_{\partial}=2 \Delta_{\bar{\partial}} .
$$

As a consequence of these facts, one gets the famous Hodge decomposition theorem:

Theorem 4 (The Hodge decomposition theorem) If a complex manifold $X$ is compact and Kähler (that is, admits a Kähler metric), then for any integer $k$, there is a (natural) decomposition into a direct sum of complex subspaces

$$
H^{k}(X, \mathbb{C})=\bigoplus_{p+q=k} H^{p, q}(X)
$$

where $H^{p, q}(X) \subset H^{k}(X, \mathbb{C})$ was defined above. 
This theorem uses the representation of cohomology classes by harmonic forms, together with the decomposition of harmonic forms. It is a remarkable fact that the final decomposition on cohomology is in fact metric independent.

- Hodge symmetry. It is clear by definition that $\overline{H^{p, q}(X)}=H^{q, p}(X)$. This is because the complex conjugate of a closed form of type $(p, q)$ is a closed form of type $(q, p)$. Here the complex conjugation acts on $H^{k}(X, \mathbb{C})$ which is the complexification of the real cohomology space $H^{k}(X, \mathbb{R})$.

In the sequel, we will rather consider the Hodge filtration defined by

$$
F^{l} H^{k}(X, \mathbb{C})=\oplus_{p \geq l} H^{p, k-p}(X) .
$$

This data is equivalent to the data of the Hodge decomposition, because by Hodge symmetry, we recover $H^{p, q}(X)$ as

$$
H^{p, q}(X)=F^{p} H^{p+q}(X, \mathbb{C}) \cap \overline{F^{q} H^{p+q}(X, \mathbb{C})},
$$

but it has the great advantage over the Hodge decomposition that it varies holomorphically, and even algebraically (see section 3) with the complex structure.

- Hodge structures. The space $H^{k}(X, \mathbb{C})$ is isomorphic to

$$
H^{k}(X, \mathbb{Z}) \otimes \mathbb{C}
$$

(where we use here Betti cohomology, that is cohomogy with value in the constant sheaves $\mathbb{Z}$ or $\mathbb{C}$, instead of de Rham cohomology).

Thus inside complex cohomology, we have on one hand integral (mod. torsion) or rational cohomology, which come from topology, and on the other hand the Hodge decomposition (it would be better to speak of the Hodge filtration at this point), which comes from complex geometry, and as we will see later on, from algebraic geometry when $X$ is complex projective, via the comparison theorems of Grothendieck-Serre. All these data together define a Hodge structure of weight $k$.

\subsection{Hodge classes and the Hodge conjecture}

Let $X$ be compact Kähler; we have the Hodge decomposition on $H^{2 k}(X, \mathbb{C})$, and make the following definitions:

Definition 5 An integral Hodge class of degree $2 k$ on $X$ is a class $\alpha \in H^{2 k}(X, \mathbb{Z})$ whose image in $H^{2 k}(X, \mathbb{C})$ is of type $(k, k)$. We will denote the group of such classes by $\operatorname{Hdg}^{2 k}(X, \mathbb{Z})$.

One defines similarly the space of rational Hodge classes by

$$
H d g^{2 k}(X):=H^{2 k}(X, \mathbb{Q}) \cap H^{k, k}(X) .
$$

The simplest examples of Hodge classes are given by the cohomology classes of closed analytic subspaces $Z \subset X$ of codimension $k$. The singular locus $Z_{\text {sing }}$ of such a $Z$ is then a closed analytic subset of $X$ which has codimension $\geq k+1$ and thus real codimension $\geq 2 k+2$. Thus one can define

$$
[Z] \in H^{2 k}(X, \mathbb{Z})
$$


by taking the cohomology class $\left[Z \backslash Z_{\text {sing }}\right] \in H^{2 k}\left(X \backslash Z_{\text {sing }}, \mathbb{Z}\right)$ of the closed complex submanifold

$$
Z \backslash Z_{\text {sing }} \subset X \backslash Z_{\text {sing }}
$$

and by observing that $H^{2 k}\left(X \backslash Z_{\text {sing }}, \mathbb{Z}\right) \cong H^{2 k}(X, \mathbb{Z})$.

The class $[Z]$ is an integral Hodge class. This can be seen using Lelong's theorem, showing that the current of integration over $Z \backslash Z_{\text {sing }}$ is well defined and closed, with cohomology class equal to the image of $[Z]$ in $H^{2 k}(X, \mathbb{C})$. On the other hand, this current anihilates all forms of type $(p, q), p \neq q, p+q=2 n-2 k, n=\operatorname{dim} X$, and it follows dually that its class is of type $(k, k)$.

The Hodge conjecture asks the following:

Conjecture 6 Let $X$ be a complex projective manifold. Then the space $H d g^{2 k}(X)$ of degree $2 k$ rational Hodge classes on $X$ is generated over $\mathbb{Q}$ by classes $[Z]$ constructed above.

Remark 7 By a well-known theorem of Chow, later on generalized by Serre, closed analytic subsets of a complex projective manifold are algebraic.

The conjecture in this form is the result of several corrections of the original conjecture (see [23]). The original conjecture concerned integral cohomology classes, and we will say something about this aspect in section 2 . In that section, we will explain further restrictions related to complex cobordism, and discovered by Atiyah and Hirzebruch, on cohomology classes of complex analytic subsets.

Furthermore, it concerned more generally the so-called Hodge level of cohomology classes, and in this generality, it was disproved by Grothendieck in [21]. Note however that the argument of Grothendieck is in fact not trivial at all, as it uses a deep result by Deligne [9] involving mixed Hodge structures and strictness of morphisms between them.

The conclusion of the paper by Grothendieck is the formulation of the generalized Hodge-Grothendieck conjecture, involving sub-Hodge structures of non maximal Hodge level, a particular case of which is conjecture 6 above.

\subsection{Lefschetz theorem on $(1,1)$-classes}

This theorem proves conjecture 6 for $k=1$, but as it proves much more, it is somewhat not representative of the general situation. First of all, it proves the Hodge conjecture also for integral Hodge classes of degree 2. Secondly, it proves a version, that we shall explain in section 1, of the Hodge conjecture extended to the compact Kähler situation, thus suggesting that an adequate generalization of the Hodge conjecture to the Kähler setting could be true. This generalization involves the most general construction of Hodge classes via analytic geometry, that is using Chern classes of coherent sheaves. We shall show in that section that this extension does not work, that is, there exist Hodge classes on compact Kähler manifolds that we cannot construct by analytic geometry methods.

The modern proof of Lefschetz theorem on $(1,1)$-classes involves the so-called exponential exact sequence, together with the Serre GAGA principle [33], guaranteing that holomorphic line bundles are algebraic on projective complex manifolds, hence admit non zero rational sections. 


\subsection{Organisation of the paper}

In the next section we will explain the impossibility to extend the Hodge conjecture to the compact Kähler case, following [38]. The example we use is in fact due to Weil in the algebraic geometry context, and we just consider his construction in the general Kähler case. The argument used here to show that the Weil Hodge classes cannot be constructed by methods of analytic geometry, is based on the Uhlenbeck-Yau theorem and its generalization by Bando and Siu, which provide further restrictions on Chern classes of coherent sheaves on compact Kähler manifolds.

In section 2, we will explain several interesting counterexamples to the Hodge conjecture for integral Hodge classes, due to Atiyah-Hirzebruch [4], Totaro [36], (topological), and Kollár [28] (non topological). The example by Kollár shows that the pathology here is very serious.

We will also discuss for particular $X$ (namely rationally connected varieties) the Hodge conjecture for integral Hodge classes and its interest with respect to the rationality problem [41].

The last section considers the problem from a much more algebro-geometric point of view. We will explain the best evidence found up to now for the Hodge conjecture, namely the algebraicity of Hodge loci due to Cattani, Deligne and Kaplan [11], and some refinements obtained in [42] of their results, exploring the question whether Hodge loci are defined over the algebraic closure of $\mathbb{Q}$, a necessary condition for the Hodge conjecture to hold true, and answering partially the following question, asked to us by V. Maillot and C. Soulé:

Question. Can the Hodge conjecture be reduced to the case of varieties defined over $\overline{\mathbb{Q}}$ ?

\section{The analytic side : Hodge classes and coherent sheaves on Kähler manifolds}

\subsection{Several constructions of Hodge classes}

- Chern classes of holomorphic vector bundles. If $E$ is a complex vector bundle on a topological manifold $X$, we have the rational Chern classes $c_{i}(E) \in$ $H^{2 i}(X, \mathbb{Q})$. (Note that the Chern classes are usually defined as integral cohomolgy classes, $c_{i} \in H^{2 i}(X, \mathbb{Z})$, but in this text, the notation $c_{i}$ will be used for the rational ones.) If $E$ is now a holomorphic vector bundle on a complex manifold $X$, the Chern classes of $E$ are Hodge classes.

This follows indeed from Chern-Weil theory, which provides de Rham representatives of $c_{i}(E)$ as follows : If $\nabla$ is a complex connection on $E$, with curvature operator $R_{\nabla} \in A_{X}^{2} \otimes E n d E$, then a representative of $c_{k}(E)$ is given by the degree $2 k$ closed form

$$
\sigma_{k}\left(\frac{i}{2 \pi} R_{\nabla}\right)
$$

where $\sigma_{k}$ is the polynomial invariant under conjugation on the space of matrices, which to a matrix associates the $k$-th symmetric function of its eigenvalues.

Now, if $E$ is a holomorphic vector bundle on $X$, there exists a complex connection $\nabla$ on $E$ such that $R_{\nabla}$ is of type $(1,1)$, that is $R_{\nabla} \in A_{X}^{1,1} \otimes E n d E$. (Given a Hermitian 
metric $h$ on $E$, one can take the so-called Chern connection, which is compatible with $h$, and has the property that its $(0,1)$-part is equal to the $\bar{\partial}$-operator of $E$.) This implies that $\sigma_{k}\left(\frac{i}{2 \pi} R_{\nabla}\right) \in A^{k, k}(X)$, and shows that $c_{k}(E)$ is Hodge.

- Chern classes of coherent sheaves. Coherent sheaves $\mathcal{F}$ on a complex manifold $X$ are sheaves of $\mathcal{O}_{X}$-modules which are locally presented as quotients

$$
\mathcal{O}_{X}^{r} \stackrel{\phi}{\rightarrow} \mathcal{O}_{X}^{s} \rightarrow \mathcal{F} \rightarrow 0
$$

where $\phi$ is a matrix of holomorphic functions. A holomorphic vector bundle gives a coherent sheaf defined as the sheaf of its local holomorphic sections. As the two data are equivalent, the vector bundle and the corresponding sheaf are often identified. Coherent sheaves associated to holomorphic vector bundles have the particularity that they are locally free, that is locally isomorphic to $\mathcal{O}_{X}^{s}$, as one sees by taking local trivializations of the considered vector bundle. A locally free analytic coherent sheaf $\mathcal{F}$ on $X$ being the sheaf of sections of a holomorphic vector bundle $F$ on $X$, we can define its Chern classes by $c_{i}(\mathcal{F}):=c_{i}(F)$.

If $X$ is a smooth projective complex manifold of dimension $n$, it is known (cf [33], [34]) that coherent sheaves on $X$ admit finite locally free resolutions

$$
0 \rightarrow \mathcal{F}_{n} \rightarrow \ldots \rightarrow \mathcal{F}_{0} \rightarrow \mathcal{F} \rightarrow 0
$$

where the $\mathcal{F}_{i}$ are locally free. We can thus define the Chern classes of $\mathcal{F}$ by the Whitney formula :

$$
c(\mathcal{F}):=\Pi_{l} c\left(\mathcal{F}_{l}\right)^{\epsilon_{l}} .
$$

In this formula, $\epsilon_{l}:=(-1)^{l}$, the total Chern class $c(\mathcal{F})$ determines the Chern classes $c_{i}(\mathcal{F})$ by the formula

$$
c(\mathcal{F})=1+c_{1}(\mathcal{F})+\ldots+c_{n}(\mathcal{F}),
$$

and the series can be inverted because the cohomology ring is nilpotent in degree $>0$. The Whitney formula and the case of holomorphic vector bundles imply that the right hand side of (1.2) is independent of the choice of locally free resolution and that the Chern classes $c_{i}(\mathcal{F})$ so defined are Hodge classes.

On a general compact complex manifold (and even Kähler), such a finite locally free resolution does not exist in general (see section 1.4), except in dimension 2 (cf [32]). In order to define the $c_{i}(\mathcal{F})$, one can use a finite locally free resolution

$$
0 \rightarrow \mathcal{F}_{n} \rightarrow \ldots \rightarrow \mathcal{F}_{0} \rightarrow \mathcal{F} \otimes \mathcal{H}_{X} \rightarrow 0
$$

of $\mathcal{F} \otimes \mathcal{H}_{X}$ by sheaves of locally free $\mathcal{H}_{X}$-modules, where $\mathcal{H}_{X}$ is the sheaf of real analytic complex functions. The $\mathcal{F}_{l}$ are then the sheaves of real analytic sections of some complex vector bundles $F_{l}$ of real analytic class, and one can then define (using again the definition $c\left(\mathcal{F}_{l}\right)=c\left(F_{l}\right)$ )

$$
\left.c(\mathcal{F})=c\left(\mathcal{F} \otimes \mathcal{H}_{X}\right), c\left(\mathcal{F} \otimes \mathcal{H}_{X}\right)\right)=\Pi_{l} c\left(\mathcal{F}_{l}\right)^{\epsilon_{l}} .
$$

This defines unambiguously the Chern classes of $\mathcal{F}$, and some further work allows to show that these classes are Hodge classes. 
The construction of rational Hodge classes as rational combinations of Chern classes of coherent sheaves is more general than the two previously given constructions. Namely, it is obvious that it generalizes Chern classes of holomorphic vector bundles, as a coherent sheaf is a more general object than a holomorphic vector bundle, but it is also true that it also generalizes the construction of classes of analytic subsets, for the following reason:

If $Z \subset X$ is a closed analytic subset of codimension $k$, then its ideal sheaf $\mathcal{I}_{Z}$ is a coherent sheaf, and one has:

$$
c_{k}\left(\mathcal{I}_{Z}\right)=(-1)^{k}(k-1) ![Z] .
$$

In the projective case, it is known that the three constructions generate over $\mathbb{Q}$ the same space of Hodge classes (cf [33] and [8]).

In the general compact Kähler case, it is a classical fact that the first and second constructions do not generate over $\mathbb{Q}$ the same space of Hodge classes. The simplest example is provided by a complex torus $T$ admitting a holomorphic line bundle $\mathcal{L}$ of indefinite curvature. This means that a de Rham representative of $c_{1}(\mathcal{L})$ is given by a real $(1,1)$-form with constant coefficients on $T$, such that the corresponding Hermitian form is indefinite. If the torus $T$ satisfying this condition is chosen general enough, its space $H d g^{2}(T)$ will be generated by $c_{1}(\mathcal{L})$, as one shows by a deformation argument. It follows that $T$ will not contain any analytic hypersurface, hence no non zero degree 2 Hodge class can be constructed as the class of a codimension 1 closed analytic subset; indeed, the cohomology class $[Z]$ of an analytic hypersurface in a torus can be represented by a non zero $(1,1)$-form with constant coefficients, whose associated Hermitian form is semi-positive. Thus the class $[Z]$ could not be a multiple of $c_{1}(\mathcal{L})$. Thus we are in a situation where a Hodge classe can be constructed as the Chern class of a holomorphic vector bundle, but not as a combination with rational coefficients of classes of closed analytic subsets.

The fact that Chern classes of coherent sheaves allow to construct strictly more Hodge classes than Chern classes of holomorphic vector bundles was proved in [38] and will be discussed in section 1.4. This is something which cannot be detected in degree 2 , as the exponential exact sequence

$$
0 \rightarrow \mathbb{Z} \rightarrow \mathcal{O}_{X} \stackrel{\exp 2 i \pi}{\rightarrow} \mathcal{O}_{X}^{*} \rightarrow 0
$$

induces:

$$
\ldots H^{1}\left(X, \mathcal{O}_{X}^{*}\right) \stackrel{c_{1}}{\rightarrow} H^{2}(X, \mathbb{Z}) \rightarrow H^{2}\left(X, \mathcal{O}_{X}\right) \ldots
$$

The group $H^{1}\left(X, \mathcal{O}_{X}^{*}\right)$ is isomorphic to the group of isomorphism classes of holomorphic line bundles. The kernel $\operatorname{Ker}\left(H^{2}(X, \mathbb{Z}) \rightarrow H^{2}\left(X, \mathcal{O}_{X}\right)\right)$ identifies to the space of integral Hodge classes of degree 2. Hence in degree 2, Chern classes of holomorphic line bundles generate all integral Hodge classes, a fact which is known as the Lefschetz theorem on $(1,1)$-classes.

Remark 8 We could also consider products $c_{i}(\mathcal{F}) c_{k-i}(\mathcal{G})$ of Chern classes of coherent sheaves. However, the Whitney formula applied to sums of copies of $\mathcal{F}$ and $\mathcal{G}$ shows that they can be expressed as rational combinations of Chern classes $c_{k}(\mathcal{H})$ of coherent sheaves $\mathcal{H}$ on $X$. 
We just saw that the most general way to construct via analytic geometry Hodge classes on Kähler manifolds is via Chern classes of coherent sheaves, since the two other methods do not provide enough Hodge classes.

If we want to extend the Hodge conjecture to the Kähler case, we therefore are led to consider the following question:

Question 9 Are Hodge classes on compact Kähler manifolds generated over $\mathbb{Q}$ by Chern classes of coherent sheaves?

The answer to this question is "no", as we proved in [38]. The two next subsections will be devoted to explaining why.

\subsection{Weil tori and Weil classes}

The Hodge classes described below have been constructed by Weil in the case of algebraic tori, as a potential counterexample to the Hodge conjecture for algebraic varieties. In the case of a general complex torus, the construction is still simpler. These complex tori have been also considered in [43] by Zucker. In the application, it will suffice to consider 4-dimensional Weil tori, but the general construction is not more complicated.

We start with a $\mathbb{Z}[I]$-action on $\Gamma:=\mathbb{Z}^{4 n}$, where $I^{2}=-1$, which makes

$$
\Gamma_{\mathbb{Q}}:=\Gamma \otimes \mathbb{Q}
$$

into a $K$-vector space, where $K$ is the quadratic field $\mathbb{Q}[I]$.

Let

$$
\Gamma_{\mathbb{C}}=\Gamma \otimes \mathbb{C}=\mathbb{C}_{i}^{2 n} \oplus \mathbb{C}_{-i}^{2 n}
$$

be the associated decomposition into eigenspaces for $I$. A $2 n$-dimensional complex torus $X$ with underlying lattice $\Gamma$ and inheriting the $I$-action is determined by a $2 n$ dimensional complex subspace $W$ of $\Gamma_{\mathbb{C}}$, which has to be stable under $I$, hence has to be the direct sum

$$
W=W_{i} \oplus W_{-i}
$$

of its intersections with $\mathbb{C}_{i}^{2 n}$ and $\mathbb{C}_{-i}^{2 n}$. It has furthermore to satisfy the condition that

$$
W \cap \Gamma_{\mathbb{R}}=\{0\} .
$$

Given $W, X$ is given by the formula

$$
X=\Gamma_{\mathbb{C}} /(W \oplus \Gamma) .
$$

We will choose $W$ so that

$$
\operatorname{dim} W_{i}=\operatorname{dim} W_{-i}=n .
$$

Then $W$, hence $X$ is determined by the choice of the $n$-dimensional subspaces

$$
W_{i} \subset \mathbb{C}_{i}^{2 n}, W_{-i} \subset \mathbb{C}_{-i}^{2 n},
$$

which have to be general enough so that the condition (1.4) is satisfied. 
We have isomorphisms

$$
H^{2 n}(X, \mathbb{Q}) \cong H_{2 n}(X, \mathbb{Q}) \cong \bigwedge^{2 n} \Gamma_{\mathbb{Q}}
$$

Consider the subspace

$$
\bigwedge_{K}^{2 n} \Gamma_{\mathbb{Q}} \subset \bigwedge^{2 n} \Gamma_{\mathbb{Q}}
$$

Since $\Gamma_{\mathbb{Q}}$ is a $2 n$-dimensional $K$-vector space, $\bigwedge_{K}^{2 n} \Gamma_{\mathbb{Q}}$ is a one dimensional $K$-vector space, and its image under this inclusion is thus a 2 dimensional $\mathbb{Q}$-vector space. The claim is that, under the assumption (1.5), $\bigwedge_{K}^{2 n} \Gamma_{\mathbb{Q}}$ is made of Hodge classes, that is, is contained in the subspace $H^{n, n}(X)$ of the Hodge decomposition. Notice that under the isomorphisms (1.6), tensored by $\mathbb{C}, H^{n, n}(X)$ identifies with the image of

$$
\bigwedge^{n} W \otimes \bigwedge^{n} \bar{W}
$$

in $\bigwedge^{2 n} \Gamma_{\mathbb{C}}$.

To prove this claim, note that we have the decomposition

$$
\Gamma_{K}:=\Gamma_{\mathbb{Q}} \otimes K=\Gamma_{K, i} \oplus \Gamma_{K,-i}
$$

into eigenspaces for the $I$ action. Then $\bigwedge_{K}^{2 n} \Gamma_{\mathbb{Q}} \subset \bigwedge^{2 n} \Gamma_{\mathbb{Q}}$ is defined as the image of $\bigwedge_{K}^{2 n} \Gamma_{K, i} \subset \bigwedge_{K}^{2 n} \Gamma_{K}$ via the trace map

$$
\bigwedge_{K}^{2 n} \Gamma_{K}=\bigwedge_{\mathbb{Q}}^{2 n} \Gamma_{\mathbb{Q}} \otimes K \rightarrow \bigwedge^{2 n} \Gamma_{\mathbb{Q}} .
$$

Now we have the inclusion

$$
\Gamma_{K} \subset \Gamma_{\mathbb{C}},
$$

with $\Gamma_{\mathbb{C}}=\Gamma_{K} \otimes_{\mathbb{Q}} \mathbb{R}$, (because $\mathbb{C} \cong K \otimes_{\mathbb{Q}} \mathbb{R}$, ) and the equality

$$
\Gamma_{K, i}=\Gamma_{K} \cap \mathbb{C}_{i}^{2 n} .
$$

The space $\Gamma_{K, i}$ is a $2 n$ dimensional $K$-vector space which generates over $\mathbb{R}$ the space $\mathbb{C}_{i}^{2 n}$. It follows that the image of $\bigwedge_{K}^{2 n} \Gamma_{K, i}$ in $\bigwedge^{2 n} \Gamma_{\mathbb{C}}$ generates over $\mathbb{C}$ the line $\bigwedge^{2 n} \mathbb{C}_{i}^{2 n}$.

But we know that $\mathbb{C}_{i}^{2 n}$ is the direct sum of the two spaces $W_{i}$ and $\overline{W_{-i}}$ which are $n$-dimensional. Hence

$$
\bigwedge^{2 n} \mathbb{C}_{i}^{2 n}=\bigwedge^{n} W_{i} \otimes \bigwedge^{n} \overline{W_{-i}}
$$

is contained in $\bigwedge^{n} W \otimes \bigwedge^{n} \bar{W}$, that is in $H^{n, n}(X)$. 


\subsection{The main result}

We proved in [38] that the Weil Hodge classes on general Weil tori provide a counterexample to question 9 , thus showing that the projectivity assumption is crucial in the statement of the Hodge conjecture.

Theorem 10 Let $T$ be a general Weil torus of dimension 4. Then any coherent sheaf $\mathcal{F}$ on $T$ satisfies :

$$
c_{2}(\mathcal{F})=0 .
$$

Thus the Weil Hodge classes constructed in the previous sections are not in the space generated by Chern classes of coherent sheaves.

The proof uses the Uhlenbeck-Yau theorem [37], and can be even shortened by using the Bando-Siu theorem [5], which extends Uhlenbeck-Yau theorem to the case of reflexive coherent sheaves. It will prove in fact that a minimal reflexive sheaf $\mathcal{F}$ on such a torus $T$ is a flat holomorphic vector bundle (by minimal, we mean "not containing any non trivial subsheaf of smaller rank"). The Uhlenbeck-Yau theorem states the existence of Hermite-Einstein metric (relative to a Kähler metric $\omega$ on a compact Kähler manifold $X$ ) on the $\omega$-stable vector bundles on $X$. In the coherent case, Bando and Siu work with singular such metrics with $L^{2}$-integrability conditions.

The stability condition for a torsion free coherent sheaf $\mathcal{F}$ on $X$ is the condition that for any subsheaf $\mathcal{G} \subset \mathcal{F}$, with the property that $0<r k \mathcal{G}<r k \mathcal{F}$, we have

$$
\frac{c_{1}(\mathcal{G}) \cdot[\omega]^{n-1}}{r k \mathcal{G}}<\frac{c_{1}(\mathcal{F}) \cdot[\omega]^{n-1}}{r k \mathcal{F}},
$$

where $n=\operatorname{dim} X$ and the intersection is the intersection pairing on cohomology between $H^{2}(X, \mathbb{R})$ and $H^{2 n-2}(X, \mathbb{R})$.

In particular, it follows from this definition that any coherent torsion free sheaf which admits no non zero subsheaf of smaller rank is stable.

Let us recall what the Hermite-Einstein condition is, at least for a non singular Hermitian metric $h$ on a holomorphic vector bundle $E$ on $X$. The Hermitian metric $h$ determines a unique complex connection $\nabla_{h}: C^{\infty}(E) \rightarrow A_{X}^{1}(E)$ on $E$, (where $A_{X}^{1}(E)$ denotes the space of $C^{\infty} 1$-forms on $X$ with values in $E$,) which has the properties that its $(0,1)$-part $\nabla_{h}^{0,1}$ is equal to the $\bar{\partial}$-operator of $E$, and that $h$ is flat with respect to $\nabla_{h}$. The Hermite-Einstein condition concerns the curvature operator $R_{\nabla_{h}}$ of this connection. It says that

$$
R_{\nabla_{h}}=\mu \omega I d_{E}+R_{\nabla_{h}}^{0},
$$

where $\omega$ is the Kähler form on $X, \mu$ is a complex coefficient which depends only on $c_{1}(E)$, and $R_{\nabla_{h}}^{0}$, which is seen as an endomorphism of $E$ whose coefficients are 2 -forms on $X$, has primitive coefficients. We recall here that a 2 -form $\alpha$ on $X$ is primitive (with respect to the given Kähler form $\omega$ ) if it satisfies:

$$
\alpha \wedge \omega^{n-1}=0 .
$$

If furthermore the form $\alpha$ is of type $(1,1)$, the primitivity implies that

$$
* \alpha=-\alpha \wedge \frac{\omega^{n-2}}{(n-2) !}
$$


where $*$ is the Hodge operator of the underlying Riemannian metric (see [40], I, 5.1).

We refer to [5] for the extension of this definition to the case of a reflexive coherent sheaf, where the metric $h$ has singularities.

Now the key point is the fact that in the examples constructed in the previous section, namely the general Weil tori $T$ of dimension 4 , we have the following properties:

1. $H d g^{2}(T)=0$.

It follows that any coherent sheaf $\mathcal{F}$ on $T$ satisfies $c_{1}(\mathcal{F})=0$. Thus, if $\mathcal{F}$ is $\omega$-stable and reflexive, in the definition (1.7) of a Hermite-Einstein metric on $\mathcal{F}$, we have $\mu=0$, and then the curvature operator satisfies by (1.8):

$$
* R_{\nabla_{h}}=-R_{\nabla_{h}} \wedge \frac{\omega^{2}}{2} .
$$

Combined with the fact that $h$ is parallel with respect to $\nabla_{h}$, we get:

$$
\left|R_{\nabla_{h}}\right|^{2} V o l=\left(\operatorname{Tr} R_{\nabla_{h}}^{2}\right) \wedge \frac{\omega^{2}}{2} .
$$

2. $T$ does not contain positive dimensional proper analytic subsets.

Recall that we want to prove $c_{2}(\mathcal{F})=0$ for any coherent sheaf $\mathcal{F}$ on $T$. Property 2 tells us that we can always reduce to the case of reflexive sheaves. Indeed any sheaf differs from its reflexive hull by torsion sheaves, and torsion sheaves must be supported on points, which cannot contribute to $H^{4}$ (they have Chern classes only in degree $8=\operatorname{dim}_{\mathbb{R}} T$ ).

Next, property 1 and the Whitney formula imply that $c_{2}$ is additive under exact sequences of coherent sheaves on such tori.

Putting together both arguments, and reasoning by induction on the rank, we only have to consider the case of a reflexive coherent sheaf $\mathcal{F}$ which does not contain any non trivial proper subsheaf of smaller rank.

We can apply Bando-Siu theorem, because this $\mathcal{F}$ is $\omega$-stable with respect to any Kähler metric $\omega$ on $X$. It turns out that we can choose $\omega$ satisfying the property that

$$
[\omega]^{2} \cdot \alpha=0,
$$

for any Weil Hodge class $\alpha$ on $T$. Note that all the Hodge classes of degree 4 on $T$ are in fact of Weil type because the complex structure on $T$ is generically chosen among the Weil complex structures. Thus we in fact have the equality

$$
[\omega]^{2} \cdot \alpha=0,
$$

for any Hodge class $\alpha$ on $T$.

We use now the computation above, leading to (a particular case of) the famous Bogomolov-Miyaoka-Yau inequality for stable bundles. Namely, integrating (1.9) over $T$, we conclude that for an Hermite-Einstein metric on a reflexive coherent sheaf $\mathcal{F}$ satisfying $c_{1}(\mathcal{F})=0$, with associated Chern connection $\nabla_{h}$, we can express the $L^{2}$-norm

$$
\left|R_{\nabla_{h}}\right|_{L^{2}}^{2}:=\int_{T}\left|R_{\nabla_{h}}\right|^{2} V o l
$$


of the associated curvature operator as an adequate multiple of

$$
c_{2}(\mathcal{F}) \cdot[\omega]^{2} .
$$

Here we use the fact that by Chern-Weil theory, an adequate linear combination of $c_{1}^{2}$ and $c_{2}$ admits as a de Rham representative the trace of the square of the curvature operator.

But we already mentioned that, in our situation

$$
[\omega]^{2} \cdot c_{2}(\mathcal{F})=0 .
$$

Thus it follows that $\left|R_{\nabla_{h}}\right|_{L^{2}}^{2}$ vanishes, and thus that $R_{\nabla_{h}}$ vanishes. Hence we conclude that $\mathcal{F}$ is locally free and that $c_{2}(\mathcal{F})=0$ (and in fact $c_{i}(\mathcal{F})=0$ for $i>0$ ).

Remark 11 We used in this sketch of proof the theorem of Bando-Siu, which is a rather hard extension of the Uhlenbeck-Yau theorem to the case of reflexive sheaves. It is possible (this is done in [38]) to avoid this in our case by working over a desingularization $\widetilde{T}$ of $T$ (at the finitely many points where $\mathcal{F}$ is not locally free) on which $\mathcal{F}$ extends as a vector bundle. Assuming as above that $\mathcal{F}$ does not contain any non trivial subsheaf of smaller rank, its locally free extension to $\widetilde{T}$ will be stable with respect to any Kähler metric and we can apply the Uhlenbeck-Yau theorem to it. This line of reasoning gives the vanishing $c_{2}(\mathcal{F})=0$ but no other information like the fact that $\mathcal{F}$ is locally free and admits a flat connection.

\subsection{Further comments on the $K$-theory of compact Kähler mani- folds}

Working a little more with the arguments described above also allows us to prove the following :

Theorem 12 [38] Let $T$ be a compact Kähler manifold of dimension $n$ satisfying the assumptions 1., 2. of the previous section and the following analogue of (1.10):

$$
\alpha \cdot[\omega]^{n-2}=0
$$

for all Hodge classes $\alpha$ of degree 4 on $T$ (for example $T$ may be a general complex torus of dimension $n \geq 3$ ). Then any holomorphic vector bundle $E$ on $T$ satisfies:

$$
c_{i}(E)=0, \forall i>0 .
$$

Remark 13 The proof gives in fact a stronger result stated in [38]. Namely, any reflexive sheaf on $T$ is in fact a flat holomorphic vector bundle, that is comes from a representation of $\pi_{1}(T)$.

As a corollary, we conclude that there exist coherent sheaves $\mathcal{F}$ on such a compact Kähler manifold which do not admit a finite locally free resolution

$$
0 \rightarrow \mathcal{F}_{n} \rightarrow \ldots \rightarrow \mathcal{F}_{0} \rightarrow \mathcal{F} \rightarrow 0
$$

(Note that it is well known that if a finite locally free resolution exists, then a finite locally free resolution of length $n$ exists.) 
Indeed, consider the ideal sheaf $\mathcal{I}_{x}$ of a point $x \in T$. If such a resolution

$$
0 \rightarrow \mathcal{F}^{n} \rightarrow \ldots \rightarrow \mathcal{F}^{i} \rightarrow \mathcal{F}^{i-1} \rightarrow \ldots \rightarrow \mathcal{F}^{0} \rightarrow \mathcal{I}_{x} \rightarrow 0
$$

would exist, then we would get the equality

$$
c\left(\mathcal{I}_{x}\right)=\Pi_{i} c\left(\mathcal{F}_{i}\right)^{\epsilon_{i}}
$$

with $\epsilon_{i}=(-1)^{i}$. But the left hand side is non zero in positive degrees since its term of degree $2 n, n=\operatorname{codim} x=\operatorname{dim} T$ is a non zero multiple of the class of $x$, by formula (1.3). On the other hand the right hand side vanishes in positive degrees by Theorem 12 .

This result shows that the $K_{0}$ group of the category of coherent sheaves on general compact Kähler manifolds may differ from the one generated by the subcategory of locally free coherent sheaves, which is not the case for smooth projective varieties (see $[8])$.

\section{The topological side: integral Hodge classes}

\subsection{Atiyah-Hirzebruch-Totaro topological obstruction.}

Atiyah and Hirzebruch [4] found counterexamples to the Hodge conjecture stated for degree $2 k$ integral Hodge classes (as opposed to rational Hodge classes), when $k \geq 2$. (We already mentioned that in degree 2 , the most optimistic statements are true, due to Lefschetz theorem.)

Recently, Totaro [36] revisited these examples of Atiyah and Hirzebruch and reformulated the obstructions they had found more directly using the complex cobordism graded ring $M U^{*}(X)$ of $X$. Let us first describe this ring which is defined for all differentiable compact manifolds : given $X$, we consider first of all the objects which are triples $(V, f, \epsilon)$, where $V$ is a differentiable compact manifold, $f: V \rightarrow X$ is a differentiable map, and $\epsilon$ is a class of a stable complex structure on the virtual normal bundle $f^{*} T_{X}-T_{V}$. Here $f^{*} T_{X}-T_{V}$ is an element of the $K_{0}$-group of real vector bundles on $V$, and choosing a stable complex structure on it means we choose an element of the $K_{0}$-group of complex vector bundles on $V$ which sends (via the natural forgetful map) to $f^{*} T_{X}-T_{V}+T$, where $T$ is the trivial real vector bundle of rank 1 or 0 .)

One now makes the following construction, which is a slight variant of the construction of the absolute complex cobordism ring $M U^{*}=M U^{*}$ (point) (see [22]): let us consider the free abelian group generated by such triples, and take its quotient by the complex cobordism relations: namely, for each differentiable compact manifold with boundary $M$, differentiable map $\phi: M \rightarrow X$, and stable complex structure $\epsilon$ on the virtual normal bundle $f^{*} T_{X}-T_{M}$, we observe that the restriction of $T_{M}$ to the boundary of $M$ is naturally isomorphic to $T_{\partial M} \oplus T$ where $T$ is trivial of rank 1. Thus the stable complex structure $\epsilon$ on the virtual normal bundle $f^{*} T_{X}-T_{M}$ induces a stable complex structure $\epsilon_{0}$ on the virtual normal bundle $f_{0}^{*} T_{X}-T_{\partial M}$, where $f_{0}$ is the restriction of $f$ to $\partial M$.

We take the quotient of the free abelian group by the relations generated by

$$
\left(\partial M, f_{0}, \epsilon_{0}\right)=0
$$




$$
\left(V_{1} \sqcup V_{2}, f_{1} \sqcup f_{2}, \epsilon_{1} \sqcup \epsilon_{2}\right)=\left(V_{1}, f_{1}, \epsilon_{1}\right)+\left(V_{2}, f_{2}, \epsilon_{2}\right) .
$$

The result will be denoted by $M U^{*}(X)$. Here the grading is given by $*=$ $\operatorname{dim} X-\operatorname{dim} V$. The product structure is given by fibered product over $X$.

Note that $M U^{*}(X)$ is naturally a $M U^{*}$-module, since elements of $M U^{*}$ is generated by data $\left(W, \epsilon_{0}\right)$ where $W$ is differentiable compact and $\epsilon_{0}$ is a stable complex structure on $T_{W}$ (here the map to a point is necessarily constant). Then for $(V, f, \epsilon)$ and $\left(W, \epsilon_{0}\right)$ as above, we can consider the product $\left(V \times W, f \circ p r_{1}, \epsilon+\epsilon_{0}\right)$.

Denote by $M U^{*}(X) \underset{M U^{*}}{\otimes} \mathbb{Z}$ its tensor product with $\mathbb{Z}$ over $M U^{*}$ (which maps by the degree to $\mathbb{Z}=H^{0}($ point, $\left.\mathbb{Z})\right)$. Thus, in $M U^{*}(X) \underset{M U^{*}}{\otimes} \mathbb{Z}$, one kills all the products $\left(V \times W, f \circ p r_{1}, \epsilon_{1}+\epsilon_{2}\right)$ where $\epsilon_{2}$ is a stable complex structure on $W$, with $\operatorname{dim} W>0$. As we have for such products

$$
\left(f \circ p r_{1}\right)_{*}\left(1_{V \times W}\right)=0,
$$

there is a natural map:

$$
\begin{gathered}
M U^{*}(X) \underset{M U^{*}}{\otimes} \mathbb{Z} \rightarrow H^{*}(X, \mathbb{Z}), \\
(V, f, \epsilon) \mapsto f_{*} 1_{V} .
\end{gathered}
$$

(Here we note that, as we have a stable complex structure on the virtual normal bundle of $f$, the Gysin image $f_{*} 1_{V}$ is well defined. If $X$ is oriented, $V$ is also naturally oriented because the virtual normal bundle of $f$ has a stable complex structure, and then $f_{*}\left(1_{V}\right)$ is the Poincaré dual cohomology class of the homology class $f_{*}\left([V]_{\text {fund }}\right)$.)

Coming back to the case where $X$ is a complex projective (or more generally compact complex) manifold, Totaro [36] observed that the cycle map

$$
\mathcal{Z}^{k}(X) \rightarrow H^{2 k}(X, \mathbb{Z}), Z \mapsto[Z],
$$

where the left hand side is the free abelian group generated by subvarieties (or irreducible closed analytic subsets) of codimension $k$ of $X$, is the composite of two maps

$$
\mathcal{Z}^{k}(X) \rightarrow\left(M U^{*}(X) \underset{M U^{*}}{\otimes} \mathbb{Z}\right)^{2 k} \rightarrow H^{2 k}(X, \mathbb{Z}) .
$$

Here the second map was introduced above, and the first map is defined using the construction of the complex cobordism ring; indeed, for any inclusion of a (maybe singular) codimension $k$ closed algebraic subset $Z \subset X$, there is a desingularization

$$
\tau: \widetilde{Z} \rightarrow X
$$

and we have

$$
[Z]=\tau_{*} 1_{\widetilde{Z}} .
$$

On the other hand, the virtual normal bundle of $\tau$ has an obvious stable complex structure. of

By the factorization $(2.11)$, a torsion class in $H^{2 k}(X, \mathbb{Z})$ which is not in the image

$$
\left(M U^{*}(X) \underset{M U^{*}}{\otimes} \mathbb{Z}\right)^{2 k} \rightarrow H^{2 k}(X, \mathbb{Z})
$$


cannot be algebraic. On the other hand, as it sends to 0 in $H^{2 k}(X, \mathbb{C})$, it is obviously an integral Hodge class.

This is a supplementary topological obstruction for an integral Hodge class to be algebraic. These obstructions are of torsion, as the map $\left(M U^{*}(X) \underset{M U^{*}}{\otimes} \mathbb{Z}\right)^{2 k} \rightarrow$ $H^{2 k}(X, \mathbb{Z})$ becomes an isomorphism when tensored by $\mathbb{Q}$. In fact they essentially concern torsion classes, as explained by Totaro, as the map

$$
\left(M U^{*}(X) \underset{M U^{*}}{\otimes} \mathbb{Z}\right)^{2 k} \rightarrow H^{2 k}(X, \mathbb{Z})
$$

is an isomorphism if $H^{*}(X, \mathbb{Z})$ has no torsion. The Atiyah-Hirzebruch example [4] shows that these obstructions are effective.

\section{$2.2 \quad$ Kollár's example}

We start this section by describing a method due to Kollár [28], which produces examples of smooth projective complex varieties $X$, together with an even degree integral cohomology class $\alpha$, which is not algebraic, that is, which is not the cohomology class of an algebraic cycle of $X$, while a non-zero multiple of $\alpha$ is algebraic. This is another sort of counterexample to the Hodge conjecture over the integers, since the class $\alpha$ is of course a Hodge class.

The examples are as follows : consider a smooth hypersurface $X \subset \mathbb{P}^{n+1}$ of degree $d$. For $l<n$, the Lefschetz theorem on hyperplane sections says that the restriction map

$$
H^{l}\left(\mathbb{P}^{n+1}, \mathbb{Z}\right) \rightarrow H^{l}(X, \mathbb{Z})
$$

is an isomorphism. Since the left-hand side is isomorphic to $\mathbb{Z} H^{k}$ for $l=2 k<n$, where $H$ is the cohomology class of a hyperplane, and is 0 for $l$ odd or $l>n$, we conclude by Poincaré duality on $X$ that for $2 k>n$, we have $H^{2 k}(X, \mathbb{Z})=\mathbb{Z} \alpha$, where $\alpha$ is determined by the condition $\left\langle\alpha, h^{n-k}\right\rangle=1$, with the notation $h=H_{\mid X}=$ $c_{1}\left(\mathcal{O}_{X}(1)\right)$. Note that the class $d \cdot \alpha$ is equal to $h^{k}$, (both have intersection number $d$ with $\left.h^{n-k}\right)$, hence is algebraic.

In the sequel, we consider for simplicity the case where $n=3, k=2$. Then $d \cdot \alpha$ is the class of a plane section of $X$.

Theorem 14 (Kollár, [28]) Assume that for some integer $p$ coprime to 6, $p^{3}$ divides $d$. Then for general $X$, any curve $C \subset X$ has degree divisible by $p$. Hence the class $\alpha$ is not algebraic.

Recall that "general" means that the defining equation for $X$ has to be chosen away from a specified union of countably many Zariski closed proper subsets of the parameter space.

Proof. Let $d=p^{3} s$, and let $Y \subset \mathbb{P}^{4}$ be a degree $s$ smooth hypersurface. Let $\phi_{0}, \ldots, \phi_{4}$ be sections of $\mathcal{O}_{\mathbb{P}^{4}}(p)$ without common zeroes. They provide a map

$$
\phi: Y \rightarrow \mathbb{P}^{4},
$$

which for a generic choice of the $\phi_{i}$ 's satisfies the following properties :

1. $\phi$ is generically of degree 1 onto its image, which is a hypersurface $X_{0} \subset \mathbb{P}^{4}$ of degree $p^{3} s=d$. 
2. $\phi$ is two-to-one generically over a surface in $X_{0}$, three-to-one generically over a curve in $X_{0}$, at most finitely many points of $X_{0}$ have more than 3 preimages, and no point has more than 4 preimages.

Let now $X \subset \mathbb{P}^{4}$ be a smooth hypersurface which is general in moduli. Let $C \subset X$ be a reduced curve. The idea is to degenerate the pair $(X, C), C \subset X$, to a pair $\left(X_{0}, C_{0}\right), C_{0} \subset X_{0}$, where $X_{0}$ was defined above. This is possible because the point parameterizing $X$ is general, and because there are only countably many relative Hilbert schemes over the moduli space of $X$, parameterizing curves in the fibers $X_{t}$. Thus a curve $C \subset X$, with $X$ general in moduli, must correspond to a point of a relative Hilbert scheme which dominates the moduli space of $X$.

By flatness, the curve $C_{0}$ has the same degree as $C$. Recall the normalization map

$$
\phi: Y \rightarrow X_{0} .
$$

By property 2 above, there exists a 1 -cycle $\tilde{z}_{0}$ in $Y$ such that $\phi_{*}\left(\tilde{z}_{0}\right)=6 z_{0}$, where $z_{0}$ is the cycle associated to $C_{0}$. It follows that

$$
6 \operatorname{deg} z_{0}=\operatorname{deg} \phi_{*}\left(\tilde{z}_{0}\right) .
$$

On the other hand, the right-hand side is equal to the degree of the line bundle $\phi^{*} \mathcal{O}_{X_{0}}(1)$ computed on the cycle $\tilde{z}_{0}$. Since $\phi^{*} \mathcal{O}_{X_{0}}(1)$ is equal to $\mathcal{O}_{Y}(p)$, it follows that this degree is divisible by $p$. Hence we found that $6 \operatorname{deg} C=6 \operatorname{deg} C_{0}=6 \operatorname{deg} z_{0}$ is divisible by $p$, and since $p$ is coprime to 6 , it follows that $\operatorname{deg} C$ is also divisible by $p$.

As remarked in [35], Kollár's example, which is not topological, exhibits the following phenomenon which illustrates the complexity of the Hodge conjecture : we can have a family $\mathcal{X} \rightarrow B$ of smooth projective complex manifolds, and a locally constant integral Hodge class $\alpha_{t} \in H^{4}\left(X_{t}, \mathbb{Z}\right)$, with the property that on a dense subset $B_{a l g} \subset B$, which is a countable union of closed proper algebraic subsets, the class $\alpha_{t}$ is algebraic, that is, is an integral combination $\left[Z_{t}\right]=\sum_{i} n_{i}\left[Z_{i, t}\right]$ of classes of codimension 2 subvarieties, but on its complementary set, which is a countable intersection of dense open subsets, the class $\alpha_{t}$ is not algebraic.

Indeed, one can show that there exists a countable union of proper algebraic subsets, which is dense for the usual topology in the parameter space of all smooth hypersurfaces of degree $d$ in $\mathbb{P}^{4}$, consisting of points parameterizing hypersurfaces $X$ for which the class $\alpha$ is algebraic. It suffices for this to prove that the set of smooth surfaces of degree $d$ in $\mathbb{P}^{3}$ carrying an algebraic class $\lambda \in H^{2}(S, \mathbb{Z}) \cap H^{1,1}(S)$ satisfying the property that $\left\langle\lambda, c_{1}\left(\mathcal{O}_{S}(1)\right)>_{S}\right.$ is coprime to $d$, is dense in the space of all surfaces of degree $d$ in $\mathbb{P}^{3}$. Indeed, for any $X$ containing such a surface, the class $\alpha$ is algebraic on $X$.

Now this fact follows from the density criterion for the Noether-Lefschetz locus explained in [40] II, 5.3.4, and from the fact that rational classes $\nu \in H^{2}(S, \mathbb{Q})$ such that a multiple $b \nu$ is integral, and satisfies $\left\langle b \nu, c_{1}\left(\mathcal{O}_{S}(1)\right)>_{S}=a\right.$ with $a$ coprime to $d$, are dense in $H^{2}(S, \mathbb{Q})$.

\subsection{Rationally connected varieties and the rationality problem}

A longstanding problem in algebraic geometry is the characterization of rational varieties, namely those smooth projective $X$ which are birationally equivalent to 
$\mathbb{P}^{n}, n=\operatorname{dim} X$.

Beautiful obstructions to rationality, very efficient in dimension three, even for unirational varieties, (for which there exists a surjective rational map $\phi: \mathbb{P}^{n} \rightarrow X$,) have been found in the papers [12], [3], [24].

In higher dimensions, the criteria above, and especially those of [12], [3] are less useful. In [35], [41], we observed that if $X$ is a smooth projective variety which is birational to $\mathbb{P}^{n}$, then the Hodge conjecture holds for integral Hodge classes on $X$ of degree $2 n-2$ and 4 . This is optimal, because in other degrees, we can blow-up a copy of Kollár's example imbedded in some projective space to get counterexamples. More generally, we have the following lemma :

Lemma 15 The groups

$$
H d g^{4}(X, \mathbb{Z}) / H d g^{4}(X, \mathbb{Z})_{a l g}, H d g^{2 n-2}(X, \mathbb{Z}) / H d g^{2 n-2}(X, \mathbb{Z})_{a l g},
$$

where the lower index "alg" means that we consider the group of integral Hodge classes which are algebraic, are both birational invariants of the complex projective variety $X$.

Proof. This follows from the resolution of singularities and the invariance under blow-ups, which is a consequence of the computation of the cohomology and the Chow groups of a blown-up variety (cf [30], or [40], I, 7.3.3, II, 9.3.3). For the degree 4 case, the new degree 4 integral Hodge classes appearing under blow-up come from degree 2 integral Hodge classes on the center of the blow-up. Hence they are algebraic by the Lefschetz theorem on $(1,1)$-classes. For the other case, the new degree $2 n-2$ integral Hodge classes appearing under blow-up of a connected smooth subvariety are multiples of the class of a line in a fiber of the blowing-down map, hence they are also algebraic.

Note the following two facts concerning rational Hodge classes of degree 4 and $2 n-2$ :

1. The Hodge conjecture (for projective varieties) is always true for rational Hodge classes of degree $2 n-2$. This is a consequence of Lefschetz theorem on $(1,1)$ classes, and of the hard Lefschetz theorem.

2. The Hodge conjecture (for projective varieties) is true for rational Hodge classes of degree 4 on varieties which are swept out by rational curves (called uniruled varieties). This is a result which is due to Conte and Murre [14] in dimension 4, and which has been generalized by Bloch and Srinivas in [7].

Thus it seems natural to consider in these situations the problem for integral Hodge classes. Starting from Kollár's example $(X, \alpha)$ in dimension 3, we can consider the product $X \times \mathbb{P}^{1}$ and either of the classes $p r_{1}^{*} \alpha, p r_{1}^{*} \alpha \cup p r_{2}^{*}$ ([point]. None of these classes is algebraic, and they are of degree 4 and $2 n-2=6$ respectively. Thus the uniruledness is not a sufficient assumption.

Rationally connected varieties, for which there passes a rational curve through any two points, have been the object of intensive work since the seminal paper by Kollár, Miyaoka and Mori [29]. Still they remain very mysterious from several points of view. They are as close as possible to unirational varieties (no example is known to be not unirational), and this is a birationally invariant class. In view of the 
birational invariance explained above, it is thus natural to consider the problem of integral Hodge classes for them:

Question 16 Let $X$ be a smooth rationally connected variety of dimension $n$. Is the Hodge conjecture true for integral Hodge classes of degree 4 or $2 n-2$ on $X$ ?

It is tempting to believe that the answer should be "yes" for degree $2 n-2$ but "no" for degree 4 , in the range $n \geq 4$.

For $n=3$, we will have the equality $4=2 n-2$, hence there is only one degree to consider. In [41], we solved this question in dimension 3 :

Theorem 17 Let $X$ be a uniruled threefold, or a regular threefold with trivial canonical bundle. Then the Hodge conjecture is satisfied by integral Hodge classes of degree 4 on $X$, that is, integral Hodge classes of degree 4 are generated by classes of curves in $X$.

The proof goes as follows: We know the Hodge conjecture for integral Hodge classes of degree 2. This is the Lefschetz theorem on $(1,1)$-classes. Let $\alpha \in H^{4}(X, \mathbb{Z})$ and let $j: \Sigma \hookrightarrow X$ be the inclusion of a smooth ample surface into $X$. The Lefschetz theorem on hyperplane restriction says that the Gysin map

$$
j_{*}: H^{2}(\Sigma, \mathbb{Z}) \rightarrow H^{4}(X, \mathbb{Z})
$$

is surjective. Assume for simplicity that $H^{2}\left(X, \mathcal{O}_{X}\right)=0$ and that $X$ is uniruled. Then there is no $H^{3,1}$-part in the Hodge decomposition of $H^{4}(X, \mathbb{C})$ and thus we want to show that any integral degree 4 cohomology class is algebraic, that is, is a combination with integral coefficients of classes of curves in $X$. Now the idea is to show that if $\Sigma$ is chosen ample enough and with the condition that

$$
\Sigma^{2} \cdot c_{1}\left(K_{X}\right)<0,
$$

then $H^{2}(\Sigma, \mathbb{Z})$ is generated over $\mathbb{Z}$ by classes which become algebraic under a small deformation of $\Sigma$ in $X$. If now $\alpha \in H^{2}(\Sigma, \mathbb{Z})$ becomes algebraic under a small deformation of $\Sigma$ in $X$, this means that for a deformation $j_{t}: \Sigma_{t} \hookrightarrow X$, the class $\alpha_{t}$ deduced from $\alpha$ by flat transport satisfies $\alpha_{t}=\sum_{i} n_{i}\left[C_{t, i}\right] \in H^{2}\left(\Sigma_{t}, \mathbb{Z}\right)$, for some curves $C_{i, t} \subset \Sigma_{t}$ and thus

$$
j_{t *} \alpha_{t}=j_{*} \alpha=\sum_{i} n_{i}\left[C_{t, i}\right] \in H^{4}(X, \mathbb{Z}),
$$

where the curves $C_{i, t}$ are now seen as curves in $X$. Thus it follows from the surjectivity of $j_{*}$ that $H^{4}(X, \mathbb{Z})$ is generated by classes of curves.

The proof that $H^{2}(\Sigma, \mathbb{Z})$ is generated over $\mathbb{Z}$ by classes which become algebraic under a small deformation of $\Sigma$ in $X$ uses the study of infinitesimal variations of Hodge structures. Using the Lefschetz theorem on $(1,1)$-classes, one has equivalently to prove that $H^{2}(\Sigma, \mathbb{Z})$ is generated over $\mathbb{Z}$ by classes which become of type $(1,1)$ under a small deformation of $\Sigma$ in $X$. Thus, this is mainly a question of showing that the spaces

$$
H^{1,1}(\Sigma)_{\mathbb{R}}:=H^{1,1}(\Sigma) \cap H^{2}(\Sigma, \mathbb{R})
$$

"move enough" with $\Sigma \subset X$ inside $H^{2}(\Sigma, \mathbb{R})$, so as to fill-in an open subset. As this open subset is a cone, it will then be clear that integral points in this cone will generate over $\mathbb{Z}$ the whole lattice $H^{2}(\Sigma, \mathbb{Z})$. 
The study of the deformations of the subspace $H^{1,1}(\Sigma)_{\mathbb{R}} \subset H^{2}(\Sigma, \mathbb{R})$ is done using Griffiths machinery of infinitesimal variations of Hodge structures for hypersurfaces [19], [40], II, 6.2.

Coming back to the question 16 , which is particularly interesting in dimension 4 , let us sketch the proof of the following result:

Theorem 18 Let $X$ be a cubic fourfold. Then any integral Hodge class on $X$ is algebraic.

Proof. The cohomology group $H^{6}(X, \mathbb{Z})$ is isomorphic to $\mathbb{Z}$ and is generated by the class of a line contained in $X$. Thus only the case of an integral Hodge class $\alpha$ of degree 4 on $X$ is to be considered. We use Zucker's method in [43], and we first recall how it works to give the result for rational Hodge classes. Thus we consider a Lefschetz pencil $\left(Y_{t}\right)_{t \in \mathbb{P}^{1}}$ of hyperplane sections of $X$, giving rise to a morphism

$$
Y \rightarrow \mathbb{P}^{1}, \tau: Y \rightarrow X
$$

where $Y$ is the blow-up of $X$ along the base-locus $\Sigma$ of the pencil. Note that the surface $\Sigma$ is a cubic surface in $\mathbb{P}^{3}$, and thus, there is a line $l \subset \Sigma$ such that for any $t \in \mathbb{P}^{1}$, there is an equality

$$
\alpha_{\mid Y_{t}}=\beta[l],
$$

where $\beta$ is an integral coefficient which does not depend on $t$. The exceptional divisor of $\tau$ is isomorphic to $\Sigma \times \mathbb{P}^{1}$. Replacing the pull-back $\tau^{*} \alpha \in H^{4}(Y, \mathbb{Z})$ by

$$
\alpha^{\prime}:=\tau^{*} \alpha-\beta\left[l \times \mathbb{P}^{1}\right],
$$

the class $\alpha^{\prime}$ satisfies now the assumption that

$$
\alpha_{\mid Y_{t}}^{\prime}=0 .
$$

Consider the family of intermediate jacobians $J \rightarrow \mathbb{P}^{1}$ with fiber

$$
J\left(Y_{t}\right):=H^{3}\left(Y_{t}, \mathbb{C}\right) /\left(F^{2} H^{3}\left(Y_{t}, \mathbb{C}\right)+H^{3}\left(Y_{t}, \mathbb{Z}\right)\right)
$$

This group identifies naturally to the kernel of the map

$$
H_{D}^{4}\left(Y_{t}, \mathbb{Z}(2)\right) \rightarrow H^{4}\left(Y_{t}, \mathbb{Z}\right) .
$$

Here the left hand side here is a Deligne cohomology group, which can be defined as the hypercohomology

$$
\mathbb{H}^{4}\left(Y_{t}, \mathbb{Z}_{D}(2)\right),
$$

where the Deligne complex $\mathbb{Z}_{D}(2)$ is the complex

$$
0 \rightarrow \mathbb{Z} \rightarrow \mathcal{O}_{Y_{t}} \rightarrow \Omega_{Y_{t}} \rightarrow 0,
$$

with $\mathbb{Z}$ put in degree 0 .

The Hodge class $\alpha^{\prime} \in H^{4}(Y, \mathbb{Z})$ provides as well a class $\alpha_{D}^{\prime} \in H_{D}^{4}(Y, \mathbb{Z}(2))$, because $H^{3}(Y, \mathbb{Z})=0$. Indeed, the Deligne complex $\mathbb{Z}_{D}(2)$ of $Y$ fits into an exact sequence:

$$
0 \rightarrow \mathcal{K} \rightarrow \mathbb{Z}_{D}(2) \rightarrow \mathbb{Z} \rightarrow 0
$$


where $\mathcal{K}$ is the complex $0 \rightarrow \mathcal{O}_{Y} \rightarrow \Omega_{Y} \rightarrow 0$ with $\mathcal{O}_{Y}$ put in degree 1 . Hence we get an exact sequence (see [40], I, 12.3)

$$
H^{3}(Y, \mathbb{Z}) \rightarrow \mathbb{H}^{4}(Y, \mathcal{K}) \rightarrow H_{D}^{4}(Y, \mathbb{Z}(2)) \rightarrow H^{4}(Y, \mathbb{Z}) .
$$

Hodge theory tells us that the image of the last map precisely consists of Hodge classes on $Y$ and that $\mathbb{H}^{4}(Y, \mathcal{K})=H^{3}(Y, \mathbb{C}) / F^{2} H^{3}(Y, \mathbb{C})$. Thus if $H^{3}(Y, \mathbb{C})=0$, then $\mathbb{H}^{4}(Y, \mathcal{K})=0$ and the last map is injective. This exact sequence also explains why we recover $J\left(Y_{t}\right)$ as the kernel of the map (2.12).

By restriction to the smooth fibers $Y_{t}$, we thus get a section

$$
\tilde{\alpha}_{D}^{\prime}(t)=\alpha_{D \mid Y_{t}}^{\prime} \in \operatorname{Ker}\left(H_{D}^{4}\left(Y_{t}, \mathbb{Z}(2)\right) \rightarrow H^{4}\left(Y_{t}, \mathbb{Z}\right)\right)=J\left(Y_{t}\right)
$$

of the family of intermediate jacobians. An essential point is the fact that this section is algebraic, for an adequate algebraic structure on $J$, compatible with the Abel-Jacobi map (cf [43]). This section is called the normal function associated to $\alpha^{\prime}$.

We know by the results of Clemens and Griffiths [12] that via the Abel-Jacobi map $\Phi_{Y_{t}}$, this fibration identifies, at least over the regular locus $U \subset \mathbb{P}^{1}$ to the fibration whose fiber over $t$ consists in rational equivalence classes of 1-cycles $z$ in $Y_{t}$ of class (or degree) $[z]=0$.

The conclusion of the paper of [43] is then obtained by saying that we can find an explicit algebraic relative family of curves $C_{s, t} \subset Y_{t}, s \in M_{t}$, such that for a generic point $t \in \mathbb{P}^{1}$, the map

$$
M_{t} \rightarrow J\left(Y_{t}\right), s \mapsto \Phi_{Y_{t}}\left(C_{s, t}-\gamma l\right), \gamma=\operatorname{deg} C_{s, t}
$$

is finite. Considering the fiber (which is finite of degree $N$ ) of this map over each $\tilde{\alpha}_{D}^{\prime}(t)$, it follows that we can find an algebraic family of curves

$$
Z_{t} \subset Y_{t}
$$

such that $\operatorname{deg} Z_{t}=N \gamma$, and

$$
\Phi_{Y_{t}}\left(Z_{t}-N \gamma l\right)=N \tilde{\alpha}_{D}^{\prime}(t) .
$$

Then the theory of the Hodge class of a normal function (see [40], II, 8.2.2) tells us that the surface $Z \subset X$ swept out by the curves $\tau\left(Z_{t}\right)$ has its class equal to $N \alpha$, modulo $[\Sigma]$. This concludes the argument of Zucker.

Unfortunately, because of this coefficient $N$ which appeared, we see that this does not solve the problem for integral Hodge classes. However, we can now use the results of Markushevitch and Tikhomirov [31], later on completed by Druel [16], which provide a generically 1-to-1 parameterization of the intermediate jacobian of $Y_{t}$ by algebraic cycles. Their result is the following

Theorem 19 Via the Abel-Jacobi map $\Phi_{Y_{t}}$, the moduli space $M_{t}$ of semi-stable rank 2 torsion free sheaves with $c_{1}=0, c_{2}=2 l$, is birational to $J\left(Y_{t}\right)$.

Here the map considered is well defined up to translation, depending on the choice of a line $l$ in $Y_{t}$, and associates to $E \in M_{t}$ the Abel-Jacobi invariant of $c_{2}(E)-2 l$, where $c_{2}(E)$ is now the refined second Chern class of $E$ with value in the Chow group $\mathrm{CH}^{2}\left(Y_{t}\right)$ of 1-cycles on $Y_{t}$ modulo rational equivalence. 
Druel makes precise the form of this birational transformation. It is the blow-up of the surface of lines of $Y_{t}$, imbedded in $J\left(Y_{t}\right)$ by the Abel-Jacobi map.

To make the above argument work, we cannot directly use the relative family $\mathcal{M}=\cup_{t \in \mathbb{P}^{1}} M_{t}$ because it does not parametrize actual curves in $Y_{t}$ but only isomorphism classes of vector bundles on $Y_{t}$. Furthermore, it is not clear if there exists an universal object over $\mathcal{M}$. However, what we can do is to build a projective bundle over $\mathcal{M}$ which parametrizes curves in $Y_{t}$.

Namely, for $k$ large enough, we consider the projective bundle over $\mathcal{M}$ whose fiber over $E_{s}$ on $Y_{t}$ is the projective space

$$
\mathbb{P}\left(H^{0}\left(Y_{t}, E_{s}(k)\right)\right) .
$$

This projective bundle $\mathbb{P}$ exists over $\mathcal{M}$, even if there is no universal object over $\mathcal{M}$, and furthermore it (or more precisely a Zariski dense open subset of it) parametrizes curves in the fibers $Y_{t}$, because for $E_{s} \in M_{t}$, a generic section $\sigma \in H^{0}\left(Y_{t}, E_{s}(l)\right)$ is transverse, so that its zero locus provides a curve $C_{s, \sigma} \subset Y_{t}$. This curve $C_{s, \sigma}$ has for rational equivalence class $c_{2}\left(E_{s}(k)\right)$, which is also equal to $c_{2}\left(E_{s}\right)$ modulo a multiple of $h^{2}, h=c_{1}\left(\mathcal{O}_{Y_{t}}(1)\right)$.

The proof of theorem 18 is now complete. Indeed, by theorem 19, our section $\tilde{\alpha}_{D}^{\prime}$ of $J$ lifts to a section of $\mathcal{M}$. As the Brauer group of a curve is trivial, this section further lifts to a section of $\mathbb{P}$, which provides a family of curves

$$
C_{t} \subset Y_{t}
$$

such that

$$
\Phi_{Y_{t}}\left(C_{t}-\beta l\right)=\tilde{\alpha}_{D}^{\prime}(t), \beta=\operatorname{deg} C_{t} .
$$

To conclude we consider, as in Zucker's proof, the surface swept out by the $C_{t}$ 's.

\section{The algebraic side : absolute Hodge classes and Hodge loci}

\subsection{Absolute Hodge classes}

Here we enter one of the most fascinating aspects of the Hodge conjecture, which seriously involves the fact that the complex manifolds we are considering are algebraic. This is related to the deep notion of Motives invented by Grothendieck, but we will focus on explaining the following essential result:

The Betti cohomology with complex coefficients of a smooth complex projective variety $X$, which a priori is of topological nature, can be computed as an algebraic invariant of $X$ seen as an algebraic variety.

Note that this is not at all true if we change the field of coefficients. Even with $\mathbb{R}$ instead of $\mathbb{C}$, and even if the variety $X$ is defined over $\mathbb{R}$, the cohomology of $X$ with real coefficients cannot be computed by algebraic means.

The meaning and the importance of this statement due to Grothendieck will be made apparent when we will explain the notion of absolute Hodge class. The key point leading to this conclusion is Serre's "GAGA principle", which says the following: 
Let $X \subset \mathbb{P}^{N}(\mathbb{C})$ be a closed algebraic subset, or more precisely a closed subscheme, and let $\mathcal{F}$ be an algebraic coherent sheaf on $X$. Sheaves here are sheaves with respect to the Zariski topology, which is much weaker than the usual topology. The typical algebraic coherent sheaves are the sheaf $\mathcal{O}_{X}$ of algebraic functions (restrictions of rational functions on $\mathbb{P}^{N}(\mathbb{C})$ with no pole on the considered open set), or ideal sheaves $\mathcal{I}_{Z}$, which are sheaves of defining equations for subschemes $Z$ of $X$. We can also consider sheaves of algebraic functions on such a $Z$, namely the quotient $\mathcal{O}_{Z}=\mathcal{O}_{X} / \mathcal{I}_{Z}$ which is considered as a sheaf of $\mathcal{O}_{X}$-modules.

Of crucial importance for us will be the sheaves of Kähler differentials $\Omega_{X / \mathbb{C}}^{l}=$ $\bigwedge^{l} \Omega_{X / \mathbb{C}}$, especially when $X$ is smooth. These sheaves have a purely algebraic definition which turns calculus into algebra (this is not surprising as we are working with polynomials). The exterior differential $d$ is naturally defined as a consequence of their construction.

Now these sheaves have analytic counterparts $\mathcal{F}^{a n}$, which are analytic coherent sheaves on the corresponding analytic subscheme $X^{a n}$ of $\mathbb{C P}^{n}$. The space of sections of the sheaf $\mathcal{F}^{a n}$ over an (usual) open set $U$ is essentially the space of sections of the sheaf $\mathcal{F}$ defined over a Zariski neighbourhood of $U$, tensored by the space of holomorphic functions on $U$. Thus morally, it is the sheaf of holomorphic sections of $\mathcal{F}$, considered in the usual topology.

The GAGA comparison theorem [33] says the following:

Theorem 20 (Serre) For any algebraic coherent sheaf $\mathcal{F}$ on $X$, one has a canonical isomorphism

$$
H^{l}(X, \mathcal{F}) \rightarrow H^{l}\left(X^{a n}, \mathcal{F}^{a n}\right),
$$

induced by the morphism of ringed spaces

$$
\phi:\left(X^{a n}, \mathcal{O}_{X^{a n}}\right) \rightarrow\left(X, \mathcal{O}_{X}\right),
$$

which satisfies : $\phi^{*} \mathcal{F}=\mathcal{F}^{\text {an }}$.

The only application we will consider concerns the sheaves $\Omega_{X / \mathbb{C}}^{l}$ of Kähler differentials. It is easy to prove that the corresponding analytic coherent sheaves are nothing but the sheaves of holomorphic differentials $\Omega_{X^{a n}}^{l}$. We thus conclude that for $X$ smooth and algebraic, and for any $p, q$, we have

$$
H^{q}\left(X, \Omega_{X / \mathbb{C}}^{p}\right) \cong H^{q}\left(X^{a n}, \Omega_{X^{a n}}^{p}\right),
$$

where on the right we have the Dolbeault cohomology of $X^{a n}$. A spectral sequence argument then allows to conclude that we have a canonical isomorphism of hypercohomology groups:

$$
\mathbb{H}^{k}\left(X, \Omega_{X / \mathbb{C}}^{\bullet} \cong \mathbb{H}^{k}\left(X^{a n}, \Omega_{X^{a n}}^{\bullet}\right) .\right.
$$

But we have the holomorphic de Rham resolution

$$
0 \rightarrow \mathbb{C} \rightarrow \mathcal{O}_{X^{a n}} \stackrel{d}{\rightarrow} \Omega_{X^{a n}} \rightarrow \ldots \rightarrow \Omega_{X^{a n}}^{n} \rightarrow 0, n=\operatorname{dim} X
$$

of the constant sheaf $\mathbb{C}$ on $X^{a n}$, which makes the constant sheaf $\mathbb{C}$ on $X^{a n}$ quasiisomorphic to the holomorphic de Rham complex $\Omega_{X^{\circ}{ }^{a n}}$. This implies that (3.13) can be written as

$$
\mathbb{H}^{k}\left(X, \Omega_{X / \mathbb{C}}^{\bullet} \cong H^{k}\left(X^{a n}, \mathbb{C}\right)\right.
$$


The right hand side in (3.14) is a purely topological object built from the usual topology on $X^{a n}$. On the other hand, the left hand side is a purely algebraic object computed from the abstract algebraic variety $X$ !

Let us recall the notion of (de Rham) absolute Hodge class (cf [13]). First of all, let us make a change of definition: a Hodge class of degree $2 k$ on $X$ will be in this section a class $\alpha \in(2 i \pi)^{k} H^{2 k}(X, \mathbb{Q}) \cap H^{k, k}(X)$. The reason for this shift is the fact that we want to use the algebraic cycle class $[Z]_{a l g}$, which takes value in algebraic de Rham cohomology, and which equals $(2 i \pi)^{k}[Z]$ via the comparison Betti-de Rham of (3.14), where $[Z]$ is the topological cycle class described in the previous section.

Let $X^{a n}$ be a complex projective manifold and $\alpha \in H d g^{2 k}\left(X^{a n}\right)$ be a Hodge class. Thus $\alpha \in(2 i \pi)^{k} H^{2 k}(X, \mathbb{Q})$ and

$$
\alpha \in F^{k} H^{2 k}\left(X^{a n}, \mathbb{C}\right) \cong \mathbb{H}^{2 k}\left(X^{a n}, \Omega_{X^{a n}}^{\bullet \geq k}\right) .
$$

Here, the left hand side is given by the Hodge filtration (0.1) on the Betti cohomology of the complex manifold $X^{a n}$ and the isomorphism of (3.15) is induced by the holomorphic de Rham resolution.

As before, the right hand side in (3.15) can be computed, by GAGA principle (Theorem 20), as the hypercohomology of the algebraic variety $X$ with value in the complex of algebraic differentials:

$$
\mathbb{H}^{2 k}\left(X^{a n}, \Omega_{X^{a n}}^{\bullet \geq k}\right) \cong \mathbb{H}^{2 k}\left(X, \Omega_{X / \mathbb{C}}^{\bullet \geq k}\right) .
$$

Let us denote by $\mathcal{E}$ the set of fields embeddings of $\mathbb{C}$ in $\mathbb{C}$. For each element $\sigma$ of $\mathcal{E}$, we get a new algebraic variety $X_{\sigma}$ defined over $\mathbb{C}$, obtained from $X$ by applying $\sigma$ to the coefficients of the defining equations of $X$, and we have a similar isomorphism for $X_{\sigma}$. Note that $\sigma$ acts on points of $\mathbb{C P}^{n}$ and induces a natural maps from $X$ to $X_{\sigma}$, but that this map is not continuous in general (the only non trivial continuous automorphism of $\mathbb{C}$ is complex conjugation).

But as an algebraic variety, $X_{\sigma}$ is deduced from $X$ by applying $\sigma$, and it follows that there is a natural (only $\tau(\mathbb{C})$-linear) map between algebraic de Rham cohomology spaces:

$$
\mathbb{H}^{2 k}\left(X, \Omega_{X / \mathbb{C}}^{\bullet \geq k}\right) \rightarrow \mathbb{H}^{2 k}\left(X_{\sigma}, \Omega_{X_{\sigma} / \mathbb{C}}^{\bullet \geq k}\right) .
$$

Applying the comparison isomorphism (3.16) in the reverse way, the class $\alpha$ provides a (de Rham or Betti) complex cohomology class

$$
\alpha_{\sigma} \in \mathbb{H}^{2 k}\left(X_{\sigma}, \Omega_{X_{\sigma}}^{\bullet \geq k}\right)=F^{k} H^{2 k}\left(X_{\sigma}^{a n}, \mathbb{C}\right)
$$

for each $\sigma \in \mathcal{E}$.

Definition 21 (cf [13]) The class $\alpha$ is said to be (de Rham) absolute Hodge if $\alpha_{\sigma}$ is a Hodge class for each $\sigma$, that is $\alpha_{\sigma}=(2 i \pi)^{k} \beta_{\sigma}$, for some rational cohomology class $\beta_{\sigma} \in H^{2 k}\left(X_{\sigma}^{a n}, \mathbb{Q}\right)$.

The main reason for introducing this definition is the following:

Proposition 22 If $Z \subset X$ is a complex subvariety of codimension $k$, then $(2 i \pi)^{k}[Z] \in$ $(2 i \pi)^{k} H^{2 k}(X, \mathbb{Q})$ is an absolute Hodge class. 
The reason for this statement is the fact that the image of $(2 i \pi)^{k}[Z]$ in $\mathbb{H}^{2 k}\left(X^{a n}, \Omega_{X}\right)$ identifies (via GAGA) to the algebraic cycle class $[Z]_{\text {alg }}$ of $Z$, an element of $\mathbb{H}^{2 k}\left(X, \Omega_{X / \mathbb{C}}\right)$ which is defined algebraically using Serre duality. Thus it follows that under the map described above

$$
H^{2 k}\left(X^{a n}, \mathbb{C}\right) \rightarrow H^{2 k}\left(X_{\sigma}^{a n}, \mathbb{C}\right),
$$

the class $(2 i \pi)^{k}[Z]=[Z]_{\text {alg }}$ is sent to $\left[Z_{\sigma}\right]_{\text {alg }}=(2 i \pi)^{k}\left[Z_{\sigma}\right]$. Here $Z_{\sigma} \subset X_{\sigma}$ is deduced from $Z$ by applying the field embedding $\sigma$ to the defining equations of $Z$.

Proposition 22 shows that the Hodge conjecture splits naturally into two subconjectures, namely:

Conjecture 23 Hodge classes on smooth complex projective varieties are absolute Hodge.

Conjecture 24 Absolute Hodge classes on smooth complex projective varieties are algebraic.

In the next section, we give a geometric description of the notion of absolute Hodge classes involving the locus of Hodge classes introduced in [11]. This essentially reduces Conjecture 23 to the question whether the connected components of the locus of Hodge classes are defined over $\overline{\mathbb{Q}}$. This geometric description will be used to answer partially the following question, asked by Soulé and Maillot :

Question 25 Can the Hodge conjecture be reduced to the case of varieties defined over $\overline{\mathbb{Q}}$ ?

This question is quite natural, for the following reason : We already mentioned that any smooth complex projective variety $X$ is a fiber of a smooth projective morphism $\pi: \mathcal{X} \rightarrow T$ defined over $\mathbb{Q}$, where $T$ is quasi-projective defined over $\mathbb{Q}$. There are countably many relative Hilbert schemes defined over $\mathbb{Q}$ parameterizing subschemes of given Hilbert polynomial in the fibers of $\pi$. Of course, if one wants to work with geometrically connected varieties, one has to replace $T$ and the relative Hilbert schemes by their connected components, which are only defined over $\overline{\mathbb{Q}}$. It follows from this that any subvariety $Z \subset X$ is the fiber of a family of cycles $\mathcal{Z} \subset \mathcal{X}^{\prime}$, where $\pi^{\prime}: \mathcal{X}^{\prime} \rightarrow T^{\prime}$ is deduced from the original family by base change from a component of a relative Hilbert scheme. In particular $T^{\prime}$ and thus $\mathcal{X}^{\prime}$ are quasiprojective defined over $\overline{\mathbb{Q}}$. We can desingularize $\mathcal{X}^{\prime}$ and complete it into a smooth projective variety $\mathcal{X}^{\prime \prime}$ defined over $\overline{\mathbb{Q}}$, and we can consider the closure $\mathcal{Z}^{\prime} \subset \mathcal{X}^{\prime \prime}$ of $\mathcal{Z}$. This is a cycle in a smooth projective variety $\mathcal{X}^{\prime \prime}$ defined over $\overline{\mathbb{Q}}$ containing $X$, and the cycle class $[Z] \in H^{2 k}(X, \mathbb{Z})$ is the restriction to $X$ of

$$
\left[\mathcal{Z}^{\prime}\right] \in H^{2 k}\left(\mathcal{X}^{\prime \prime}, \mathbb{Z}\right)
$$

In other words, any cycle class is the restriction of a cycle class on a big ambient variety defined over $\overline{\mathbb{Q}}$. This makes Question 25 quite natural.

Concerning this question, we prove for example the following result:

Proposition 26 [42] If the Hodge conjecture is true for absolute Hodge classes on varieties defined over $\overline{\mathbb{Q}}$, then it is true for absolute Hodge classes on any complex projective variety. 
Note that this proposition can be rephrased by saying that Conjecture 24 concerns in fact absolute Hodge classes on varieties defined over $\overline{\mathbb{Q}}$.

As mentioned above, the proof necessitates the rewriting of the arithmetic notion of absolute Hodge class in terms of something more familiar to people working in the theory of variations of Hodge structures, namely the notion of Hodge loci which we will describe in next section.

In that section, we will also give partial answers to Conjecture 23 and to question 25 above.

Proposition 26 reduces the Hodge conjecture for absolute Hodge classes, that is Conjecture 24 , to the countably many algebraic varieties defined over $\overline{\mathbb{Q}}$, and to the countably many Hodge classes on them. On the other hand, there are a number of crucial instances of the Hodge conjecture which are not known to hold and which concern absolute Hodge classes:

1. Hodge classes on abelian varieties (by Deligne [13], they are absolute).

2. Künneth components of the diagonal of $X \times X$. (The class of the diagonal of $X$ is algebraic, but its Künneth components obtained using the Künneth decomposition of $H^{*}(X \times X)$ are absolute Hodge classes, not known to be algebraic in general.)

3. The inverses of the Lefschetz isomorphisms give rise to Hodge classes in $X \times X$ which are absolute, but not known to be Hodge classes.

The Hodge conjecture applied to examples 2 and 3 are the so-called Lefschetz conjectures, which are parts of the standard conjectures (see [25]).

\subsection{Locus of Hodge classes}

The key point in which algebraic geometry differs from Kähler geometry is the fact that a smooth complex projective variety $X$ does not come alone, but accompanied by a full family of deformations $\pi: \mathcal{X} \rightarrow T$, where $\pi$ is smooth and projective (that is $\mathcal{X} \subset T \times \mathbb{P}^{n}$ over $T$ ), and where the basis $T$ is quasi-projective smooth and defined over $\mathbb{Q}$. (Here $T$ is not supposed to be geometrically connected). Indeed, one can take for $T$ a desingularization of a Zariski open set of the reduced Hilbert scheme parameterizing subschemes of $\mathbb{P}^{n}$ with same Hilbert polynomial as $X$. The existence of this family of deformations is reflected in the transformations $X \mapsto X_{\sigma}$ considered above. Namely, the variety $T$ being defined over $\mathbb{Q}, \sigma$ acts on its complex points, and if $X$ is the fiber over some complex point $0 \in T$, then $X_{\sigma}$ is the fiber over the complex point $\sigma(0)$ of $T$.

The total space $\mathcal{X}$ is thus an algebraic variety defined over $\mathbb{Q}$ (and in fact we may even complete it to a smooth projective variety defined over $\mathbb{Q}$ ), but for the moment, let us consider it as a family of smooth complex varieties, that is, let us work with $\pi: \mathcal{X}^{a n} \rightarrow T^{a n}$.

Associated to this family are the Hodge bundles

$$
F^{l} \mathcal{H}^{k}:=R^{k} \pi_{*}\left(\Omega_{\mathcal{X}^{a n} / T^{a n}}^{\bullet \geq l}\right) \subset \mathcal{H}^{k}:=R^{k} \pi_{*}\left(\Omega_{\mathcal{X}^{a n} / T^{a n}}\right)=R^{k} \pi_{*} \mathbb{C} \otimes \mathcal{O}_{T^{a n}},
$$

which are coherent analytic locally free sheaves with respective fibers over $t \in T$

$$
F^{l} H^{k}\left(X_{t}, \mathbb{C}\right) \subset H^{k}\left(X_{t}, \mathbb{C}\right) .
$$


In (3.17), the last isomorphism

$$
R^{k} \pi_{*}\left(\Omega_{\mathcal{X}^{a n} / T^{a n}}\right)=R^{k} \pi_{*} \mathbb{C} \otimes \mathcal{O}_{T^{a n}}
$$

is induced by the resolution of the sheaf $\pi^{-1} \mathcal{O}_{T^{a n}}$ by the relative holomorphic de Rham complex.

We shall denote by $F^{l} H^{k}$ the total space of the corresponding vector bundles.

Definition 27 (cf [11]) The locus of Hodge classes for the family $\mathcal{X} \rightarrow T$ and in degree $2 k$ is the subset

$$
Z \subset F^{k} H^{2 k}
$$

consisting of classes $\alpha_{t} \in F^{k} H^{2 k}\left(X_{t}, \mathbb{C}\right) \cap(2 i \pi)^{k} H^{2 k}\left(X_{t}, \mathbb{Q}\right)$.

This locus is thus the set of all Hodge classes in fibers of $\pi$.

For $\alpha \in Z$ we shall denote by $Z_{\alpha}$ the connected component of $Z$ passing through $\alpha$ and by $T_{\alpha}$ the projection of $Z_{\alpha}$ to $T$. $T_{\alpha}$ is the Hodge locus of $\alpha$, that is the locus of deformations of $X$ where $\alpha$ deforms as a Hodge class.

Let us give a description of the locus of Hodge classes from a local point of view: we want to describe all the pairs $(t, \alpha), t \in T, \alpha \in(2 i \pi)^{k} H^{2 k}\left(X_{t}, \mathbb{Q}\right)$, such that $\alpha \in F^{k} H^{2 k}\left(X_{t}, \mathbb{C}\right)$. Let us choose a connected and simply connected neighbourhood of 0 in $T$. Then all the fibers $X_{t}, t \in U$, are canonically homeomorphic to $X_{0}$, so that each $\alpha \in(2 i \pi)^{k} H^{2 k}\left(X_{0}, \mathbb{Q}\right)$ provides a constant section $\tilde{\alpha}_{t} \in(2 i \pi)^{k} H^{2 k}\left(X_{t}, \mathbb{Q}\right), t \in$ $U$.

Thus, over any connected simply connected open neighborhood $U \subset T$ of 0 , the locus of Hodge classes splits as the countable union over all $\alpha \in(2 i \pi)^{k} H^{2 k}\left(X_{0}, \mathbb{Q}\right)$ of the sets $Z_{\alpha}:=\left\{t \in U, \tilde{\alpha}_{t} \in F^{k} H^{2 k}\left(X_{t}, \mathbb{C}\right)\right\}$. But each $Z_{\alpha}$ (seen inside $F^{k} H_{\mid U}^{2 k}$ ) is a closed analytic subset, because $\tilde{\alpha}$ is a constant, hence holomorphic, section of the bundle $H^{2 k}$ with fiber $H^{2 k}\left(X_{t}, \mathbb{C}\right)$ over $U$, that is $\tilde{\alpha} \in \mathcal{H}^{2 k}$, and $t \in Z_{\alpha}$ if and only if the projection of $\tilde{\alpha}$ in the holomorphic quotient $\mathcal{H}^{2 k} / F^{k} \mathcal{H}^{2 k}$ vanishes.

The above description is highly transcendental, as it makes use of the locally defined constant section $\tilde{\alpha}$ which is definitely not algebraic. Note now the fact that the complex (maybe reducible) manifold $F^{k} H^{2 k}$ is in fact algebraic and defined over $\mathbb{Q}$. Indeed, using GAGA principle, the coherent sheaf $F^{k} \mathcal{H}^{2 k}$ is simply the analytic coherent sheaf associated to the algebraic sheaf $R^{2 k} \pi_{*}\left(\Omega_{\mathcal{X} / T}^{\bullet}\right)$ which is defined over $\mathbb{Q}$ on $T$. (Here the $R^{2 k} \pi_{*}$ is the algebraic derived functor.)

Thinking a little more, we see that if $\sigma: \mathbb{C} \rightarrow \mathbb{C}$ is a field embedding, then $\sigma$ acts on the points of the complex manifold $F^{k} H^{2 k}$ (because it is defined over $\mathbb{Q}$ ), and that if $\left(t, \alpha_{t}\right) \in F^{k} H^{2 k}$ is a complex point of this complex manifold, then $\sigma\left(t, \alpha_{t}\right)$ is nothing but the class $\alpha_{t, \sigma} \in F^{k} H^{2 k}\left(X_{t, \sigma}^{a n}\right)$ we considered in the previous section.

In conclusion, we find the following interpretation of the notion of absolute Hodge class.

Lemma 28 To say that Hodge classes of degree $2 k$ on fibers of the family $\mathcal{X} \rightarrow T$ are absolute Hodge is equivalent to say that the locus $Z$ is a countable union of closed algebraic subsets of $F^{k} H^{2 k}$ defined over $\mathbb{Q}$. To say that $\alpha$ is an absolute Hodge class is equivalent to say that $Z_{\alpha}$ is a closed algebraic subset of $F^{k} H^{2 k}$ defined over $\overline{\mathbb{Q}}$ and that its images under $G a l(\overline{\mathbb{Q}}: \mathbb{Q})$ are again components of the locus of Hodge classes. 
Indeed, if we have an algebraic variety $Y$ which is defined over $\mathbb{Q}$, and consider a complex point $y \in Y$, then the points $y_{\sigma}$, for all fields embeddings $\sigma: \mathbb{C} \rightarrow \mathbb{C}$ fill-in a subset of the $\mathbb{Q}$-Zariski closure of $y$, which is the countable intersection of Zariski dense open sets in this $\mathbb{Q}$-Zariski closure. Hence this subset is dense (for the usual topology) in this $\mathbb{Q}$-Zariski closure. Thus if $\alpha_{\sigma}$ is absolute, we conclude that a countable intersection of Zariski dense open sets of the $\mathbb{Q}$-Zariski closure of $\alpha$ is contained in $Z$. It follows then from the local analytic description of $Z$, which shows that it is locally a countable union of closed analytic subsets, that the $\mathbb{Q}$-Zariski closure of $\alpha$ is contained in $Z$. Finally, an easy countability argument shows that for countably many adequately chosen points in $Z, Z$ is equal to the union of their $\mathbb{Q}$-Zariski closure.

Let us now state the following beautiful theorem, due to Cattani, Deligne and Kaplan:

Theorem 29 [11] The connected components $Z_{\alpha}$ of $Z$ are closed algebraic subsets of $F^{k} H^{2 k}$. As a consequence, the Hodge loci $T_{\alpha}$ are closed algebraic subsets of $T$.

This theorem is very deep. It is very much expected if one believes in the Hodge conjecture, because then the $Z_{\alpha}$ will be the images by the algebraic cycle class of universal relative cycles parameterized by components of the relative Hilbert scheme of $\mathcal{X} \rightarrow T$.

On the other hand, the local description of the components of the locus of Hodge classes given above is completely transcendental, which makes this theorem striking.

Let us now give an idea of the proof of Proposition 26. The key ingredient is the global invariant cycle theorem (Theorem 30 below) due to Deligne [9]. Let $Y$ be a smooth complex algebraic variety, and $U \subset Y$ a Zariski open set. Let $\phi: U \rightarrow B$ be a smooth proper algebraic morphism, where $B$ is quasi-projective. Thus the fibers of $\phi$ are smooth complex projective varieties and there is a monodromy action:

$$
\rho: \pi_{1}(B, 0) \rightarrow \text { Aut } H^{l}\left(Y_{0}, \mathbb{Q}\right), 0 \in B .
$$

Theorem 30 The space of invariant classes

$$
H^{l}\left(Y_{0}, \mathbb{Q}\right)^{\rho}:=\left\{\alpha \in H^{l}\left(Y_{0}, \mathbb{Q}\right), \rho(\gamma)(\alpha)=\alpha, \forall \gamma \in \pi_{1}(B, 0)\right\}
$$

is equal to the image of the restriction map (which is a morphism of Hodge structures):

$$
H^{l}(Y, \mathbb{Q}) \rightarrow H^{l}\left(Y_{0}, \mathbb{Q}\right) .
$$

In particular it is a sub-Hodge structure of $H^{l}\left(Y_{0}, \mathbb{Q}\right)$.

Let us now put everything together: let $X$ be complex projective and $\alpha \in$ $(2 i \pi)^{k} H^{2 k}(X, \mathbb{Q})$ be an absolute Hodge class. There is a smooth projective map

$$
\pi: \mathcal{X} \rightarrow T
$$

defined over $\overline{\mathbb{Q}}$, with $\mathcal{X}$ and $T$ smooth quasi-projective, and such that $X$ is the fiber of $\pi$ over a complex point of $T$ (the smooth locus of $\pi$ ).

As we explained above, the fact that $\alpha$ is absolute Hodge implies that the component of the Hodge locus containing $\alpha$, say $Z_{\alpha}$, is defined over $\overline{\mathbb{Q}}$. We consider the 
reduced subscheme underlying $Z_{\alpha}$, say $R_{\alpha}$, which we may assume by shrinking to be smooth and connected. Then we make the base change $R_{\alpha} \rightarrow T$, which gives

$$
\pi_{\alpha}: \mathcal{X}_{\alpha} \rightarrow R_{\alpha}
$$

where both varieties are smooth quasi-projective and defined over $\overline{\mathbb{Q}}$, and $\pi_{\alpha}$ is smooth projective.

Tautologically, over $R_{\alpha}$ we have a holomorphic section $\tilde{\alpha}$ of $F^{k} H^{2 k}$. By definition of $R_{\alpha}$, this holomorphic section has the particularity that at any point $t \in R_{\alpha}$, $\alpha_{t} \in(2 i \pi)^{k} H^{2 k}\left(X_{t}, \mathbb{Q}\right)$. But then, by countability, we conclude that $\tilde{\alpha}$ is a locally constant section of $H^{2 k}$, which is everywhere of type $(k, k)$. Hence our class $\alpha$ extends to a locally constant section of the local system $R^{2 k} \pi_{\alpha *} \mathbb{Q}$, which means equivalently that it is invariant under the monodromy action.

Now we introduce a smooth compactification $\overline{\mathcal{X}_{\alpha}}$ defined over $\overline{\mathbb{Q}}$. The global invariant cycles theorem tells us that there is a Hodge class

$$
\beta \in(2 i \pi)^{k} H^{2 k}\left(\overline{\mathcal{X}_{\alpha}}, \overline{\mathbb{Q}}\right) \cap F^{k} H^{2 k}\left(\overline{\mathcal{X}_{\alpha}}\right)
$$

which restricts to $\alpha$ on $X$. With some more work, one can show that $\beta$ can be chosen absolute Hodge. Now, if the Hodge conjecture is true for $\beta$, it is true for $\alpha$.

In fact the global invariant cycles theorem and the same proof as above show as well the following statement :

Proposition 31 ([42]) Let $\alpha \in F^{k} H^{2 k}$ be a Hodge class, such that the Hodge locus $T_{\alpha}$ is defined over $\overline{\mathbb{Q}}$. Then the Hodge conjecture is true for $\alpha$ if it is true for Hodge classes on varieties defined over $\overline{\mathbb{Q}}$.

The conclusion is that, to answer question 25, we only have to investigate the question whether the Hodge loci $T_{\alpha}$ are defined over $\overline{\mathbb{Q}}$, which as explained above is weaker than the question whether Hodge class are absolute.

Concerning this last problem, we conclude with the following criterion, also proved in [42]:

Theorem 32 Let $\alpha \in F^{k} H^{2 k}(X, \mathbb{C})$ be a Hodge class. Suppose that all the constant sub-Hodge structures $L \subset H^{2 k}\left(X_{t}, \mathbb{Q}\right), t \in T_{\alpha}$, are purely of type $(k, k)$. Then $T_{\alpha}$ is defined over $\overline{\mathbb{Q}}$, and its translates under $G a l(\overline{\mathbb{Q}} / \mathbb{Q})$ are again of the form $T_{\beta}$.

As a corollary, one gets the following :

Corollary 33 Suppose that the only proper sub-Hodge structures of $H^{2 k}(X, \mathbb{Q})$ are purely of type $(k, k)$ and that the infinitesimal Torelli theorem holds for the variation of Hodge structure on $H^{2 k}\left(X_{t}, \mathbb{Q}\right)$ on $T$. Then if the Hodge locus of $\alpha$ has positive dimension, it is defined over $\overline{\mathbb{Q}}$.

The assumptions in the theorem or its corollary are reasonably easy to check in practice, for example by infinitesimal methods. On the other hand, they are clearly not satisfied in a case where the component $T_{\alpha}$ of the Hodge locus consists of one isolated point, if the Hodge structure on $H^{2 k}(X)$ is not trivial. In this case, what predicts the Hodge conjecture is that this point should be defined over $\overline{\mathbb{Q}}$. But our criterion does not give this: in fact our criterion applies only when we actually have a non trivial variation of Hodge structure along $T_{\alpha}$. 
Note that Theorem 32 also addresses partially Conjecture 23, in view of Lemma 28. Indeed, we know by Theorem 29 that the $Z_{\alpha}$ 's are algebraic, and thus by Lemma 28 , Conjecture 23 is a question about the definition field of the $Z_{\alpha}$ 's and their translates under $\operatorname{Gal}(\overline{\mathbb{Q}} / \mathbb{Q})$. Theorem 32 addresses the same question for the $T_{\alpha}$ 's instead of $Z_{\alpha}$.

\section{References}

[1] Y. André. Déformation et spécialisation de cycles motivés, Journal de l'Institut de Mathématiques de Jussieu, Vol. 5, 4, 2006.

[2] Y. André. Pour une théorie inconditionnelle des motifs, Publications Mathématiques de l'IHÉS, 83 , p. 5-49 (1996).

[3] M. Artin, D. Mumford. Some elementary examples of unirational varieties which are not rational, Proc. London Math. Soc. 25 , 75-95 (1972).

[4] M.F. Atiyah, F. Hirzebruch. Analytic cycles on complex manifolds, Topology 1, $25-45$ (1962).

[5] S. Bando, Y.-T. Siu. Stable sheaves and Einstein-Hermitian metrics, in Geometry and Analysis on Complex Manifolds (T. Mabuchi et al. Eds), World Scientific, New Jersey 39-50 (1994).

[6] S. Bloch, H. Esnault. The coniveau filtration and non-divisibility for algebraic cycles, Math. Annalen 304, 303-314 (1996).

[7] S. Bloch, V. Srinivas. Remarks on correspondences and algebraic cycles. Amer. J. Math. 105, no. 5, 1235-1253 (1983).

[8] A. Borel, J.-P. Serre. Le Théorème de Riemann-Roch, Bull. Soc. Math. France, 86, 97-136 (1958).

[9] P. Deligne. Théorie de Hodge II, Publ. Math. IHES 40, 5-57 (1971).

[10] J. Carlson, P. Griffiths. Infinitesimal variations of Hodge structure and the global Torelli theorem, in Géométrie algébrique, Angers 1980, (Ed. A. Beauville), Sijthoff-Noordhoff, 51-76.

[11] E. Cattani, P. Deligne, A. Kaplan, On the locus of Hodge classes, J. Amer. Math. Soc. 8, 483-506 (1995).

[12] H. Clemens, P. Griffiths. The intermediate Jacobian of the cubic threefold, Ann. of Math. 95, 281-356 (1972).

[13] P. Deligne. Hodge cycles on abelian varieties (notes by JS Milne), in Springer LNM, 900, 9-100 (1982).

[14] A. Conte, J. Murre. The Hodge conjecture for fourfolds admitting a covering by rational curves. Math. Ann. 238, no. 1, 79-88 (1978).

[15] P. Deligne, P. Griffiths, J. Morgan, D. Sullivan. Real Homotopy Theory of Kähler Manifolds, Inventiones Math. 29, 245-274 (1975). 
[16] S. Druel. Espaces des modules des faisceaux semi-stables de rang 2 et de classes de Chern $c_{1}=0, c_{2}=2$ et $c_{3}=0$ sur une hypersurface cubique lisse de $\mathbb{P}^{4}$, IMRN 19 (2000), 985-1004.

[17] H. Esnault, K. Paranjape. Remarks on absolute de Rham and absolute Hodge cycles. C. R. Acad. Sci. Paris Sér. I Math. 319, no. 1, 67-72 (1994).

[18] W. Fulton. Intersection Theory, Ergebnisse der Mathematik und ihrer Grenzgebiete 3.Folge.Band 2, Springer-Verlag (1984).

[19] Ph. Griffiths. On the periods of certain rational integrals I,II, Ann. of Math. 90, 460-541 (1969).

[20] Ph. Griffiths. Periods of integrals on algebraic manifolds, I, II, Amer. J. Math. 90, 568-626, 805-865 (1968).

[21] A. Grothendieck. Hodge's general conjecture is false for trivial reasons, Topology $8,299-303$ (1969).

[22] F. Hirzebruch. Topological Methods in Algebraic Geometry, 3rd ed. SpringerVerlag, Berlin (1966).

[23] W.V.D. Hodge. The topological invariants of algebraic varieties, Proc. Intern. Congr. Math. (Cambridge, Mass., Aug. 30-Sept. 6, 1950) 1, 182-192 (1952).

[24] V. Iskovskikh, Y. Manin. Three dimensional quartics and counterexamples to the Lüroth problem, Math. USSR Sbornik, 141-166 (1971).

[25] S. Kleiman. Algebraic cycles and the Weil conjectures. Dix esposés sur la cohomologie des schémas, pp. 359-386. North-Holland, Amsterdam; Masson, Paris, (1968).

[26] K. Kodaira. On Kähler varieties of restricted type (an intrinsic characterization of algebraic varieties), Ann. of Math. 60, 28-48 (1954).

[27] J. Kollár. Rational curves on algebraic varieties, Ergebnisse der Math. und ihrer Grenzgebiete, Springer-Verlag (1996).

[28] J. Kollár. Lemma p. 134 in Classification of irregular varieties, edited by E. Ballico, F. Catanese, C. Ciliberto, Lecture Notes in Math. 1515, Springer (1990).

[29] J. Kollár, Y. Miyaoka, S. Mori. Rationally connected varieties. J. Algebraic Geom. 1 no. 3, 429-448 (1992).

[30] Yu. Manin. Correspondences, motifs and monoidal transformations. (Russian) Mat. Sb. (N.S.) 77 (119), 475-507 (1968).

[31] D. Markushevich, A. Tikhomirov. The Abel-Jacobi map of a moduli component of vector bundles on the cubic threefold, J. Algebraic Geometry 10 (2001), 3762 .

[32] H.-W. Schuster. Locally free resolutions of coherent sheaves on surfaces, J. Reine Angew. Math. 337, 159-165 (1982). 
[33] J.-P. Serre. Géométrie algébrique et géométrie analytique, Ann. Inst. Fourier 6 , 1-42 (1956).

[34] J.-P. Serre. Faisceaux algébriques cohérents, Ann. Math. 61, 197-278 (1955).

[35] C. Soulé, C. Voisin. Torsion cohomology classes and algebraic cycles on complex projective manifolds, Advances in Mathematics, Special volume in honor of Michael Artin, Part I, Vol 198/1 pp 107-127 (2005).

[36] B. Totaro. Torsion algebraic cycles and complex cobordism, Journal of the American Mathematical Society 10, 467-493, (1997).

[37] K. Uhlenbeck, S.-T. Yau. On the existence of Hermitian-Yang-Mills connections in stable vector bundles, Comm. Pure Appl. Math. 257-293 (1986).

[38] C. Voisin. A counterexample to the Hodge conjecture extended to Kähler varieties, IMRN , n0 20, 1057-1075 (2002).

[39] C. Voisin. On the homotopy types of compact Kähler and complex projective manifolds, Inventiones Math. Volume 157, Number 2, 329 - 343 (2004).

[40] C. Voisin. Hodge Theory and Complex Algebraic Geometry I, II, Cambridge studies in advanced Mathematics 76, 77, Cambridge University Press 2003.

[41] C. Voisin. On integral Hodge classes on uniruled and Calabi-Yau threefolds, in Moduli Spaces and Arithmetic Geometry, Advanced Studies in Pure Mathematics 45, 2006, pp. 43-73.

[42] C. Voisin. Hodge loci and absolute Hodge classes, to appear in Compositio Math. (2007).

[43] S. Zucker. The Hodge conjecture for cubic fourfolds, Compositio Math. 34, 199-209 (1977). 